\title{
Low-Cost Air Quality Sensing towards Smart Homes
}

\author{
Hamid Omidvarborna ${ }^{1}{ }^{\mathbb{D}}$, Prashant Kumar ${ }^{1,2, * \mathbb{D}}$, Joe Hayward ${ }^{1}$, Manik Gupta ${ }^{3}$ and \\ Erick Giovani Sperandio Nascimento 4 (D)
}

1 Global Centre for Clean Air Research (GCARE), Department of Civil and Environmental Engineering, Faculty of Engineering and Physical Sciences, University of Surrey, Guildford GU2 7XH, UK; h.omidvarborna@surrey.ac.uk (H.O.); j.d.hayward@surrey.ac.uk (J.H.)

2 Department of Civil, Structural \& Environmental Engineering, Trinity College Dublin, D02 PN40 Dublin, Ireland

3 Computer Science and Information Systems, BITS Pilani-Hyderabad Campus, Pilani 500078, India; manik@hyderabad.bits-pilani.ac.in

4 Post-Graduate Program on Computational Modelling and Industrial Technology, SENAI CIMATEC University Centre, 41650-010 Salvador, BA, Brazil; erick.sperandio@fieb.org.br

* Correspondence: p.kumar@surrey.ac.uk or Prashant.Kumar@cantab.net

Citation: Omidvarborna, H.; Kumar,

P.; Hayward, J.; Gupta, M.;

Nascimento, E.G.S. Low-Cost Air Quality Sensing towards Smart Homes. Atmosphere 2021, 12, 453. https://doi.org/10.3390/atmos 12040453

Academic Editor:

Theodoros Christoudias

Received: 2 March 2021

Accepted: 29 March 2021

Published: 2 April 2021

Publisher's Note: MDPI stays neutral with regard to jurisdictional claims in published maps and institutional affiliations.

Copyright: (c) 2021 by the authors. Licensee MDPI, Basel, Switzerland. This article is an open access article distributed under the terms and conditions of the Creative Commons Attribution (CC BY) license (https:// creativecommons.org/licenses/by/ $4.0 /)$.

\begin{abstract}
The evolution of low-cost sensors (LCSs) has made the spatio-temporal mapping of indoor air quality (IAQ) possible in real-time but the availability of a diverse set of LCSs make their selection challenging. Converting individual sensors into a sensing network requires the knowledge of diverse research disciplines, which we aim to bring together by making IAQ an advanced feature of smart homes. The aim of this review is to discuss the advanced home automation technologies for the monitoring and control of IAQ through networked air pollution LCSs. The key steps that can allow transforming conventional homes into smart homes are sensor selection, deployment strategies, data processing, and development of predictive models. A detailed synthesis of air pollution LCSs allowed us to summarise their advantages and drawbacks for spatio-temporal mapping of IAQ. We concluded that the performance evaluation of LCSs under controlled laboratory conditions prior to deployment is recommended for quality assurance/control (QA/QC), however, routine calibration or implementing statistical techniques during operational times, especially during longterm monitoring, is required for a network of sensors. The deployment height of sensors could vary purposefully as per location and exposure height of the occupants inside home environments for a spatio-temporal mapping. Appropriate data processing tools are needed to handle a huge amount of multivariate data to automate pre-/post-processing tasks, leading to more scalable, reliable and adaptable solutions. The review also showed the potential of using machine learning technique for predicting spatio-temporal IAQ in LCS networked-systems.
\end{abstract}

Keywords: smart homes; low-cost sensors; affordable pollution sensing; deployment strategies; machine learning; predictive modelling

\section{Introduction}

Indoor air pollution is placed among the top five environmental public health risks that cause morbidity and mortality globally. The majority of people spend more than $90 \%$ of their time in indoor environments [1,2], and health problems and diseases associated with poor indoor air quality (IAQ) can cause a variety of adverse health effects to them [3,4]. The time spent indoors recently increased significantly in year 2020 due to severe acute respiratory syndrome coronavirus 2 (SARS-CoV-2) pandemic when people are advised to 'stay home stay safe' to protect health workers $[5,6]$.

Table 1 summarises common indoor air pollutants, their sources, and current guidelines to maintain IAQ. Air pollutants inside indoor environments can be generated from different sources, including occupants' exhalation (carbon dioxide; $\mathrm{CO}_{2}$ ), activities such as cooking and smoking, emissions from building materials, etc. from which various air 
pollutants, such as carbon monoxide (CO), particulate matter (PM), and volatile organic compounds (VOCs) are released [2,7]. $\mathrm{CO}_{2}$ is not counted as an air pollutant, but its level represents the performance of ventilation systems, especially in wintertime, whereas high $\mathrm{CO}_{2}$ levels represent poor ventilation and possible accumulation of other indoor air pollutants [8-10]. Additionally, IAQ could be affected by local outdoor air pollutants, which can ingress into indoor environments (see Table 1).

Table 1. The unhealthy exposure thresholds defined for the common indoor and outdoor air pollutants [11-13].

\begin{tabular}{|c|c|c|c|}
\hline Pollutants & Indoor Air & Outdoor Air & References \\
\hline $\begin{array}{c}\text { Benzene }\left(\mathrm{C}_{6} \mathrm{H}_{6}\right) \\
{\left[\mu \mathrm{g} \mathrm{m} \mathrm{m}^{-3}\right]}\end{array}$ & $\begin{array}{l}\text { Carcinogenic compounds, no safe level of exposure } \\
\text { recommended risk of leukaemia estimated as } 6 \times 10^{-6} \\
\text { at } 1 \mu \mathrm{g} \mathrm{m}^{-3} \text {, } \\
\text { World Health Organisation (WHO). }\end{array}$ & $\begin{array}{l}5 \text { (annual) European Union (EU) } \\
1.7 \text { (annual) WHO }\end{array}$ & {$[13,14]$} \\
\hline $\begin{array}{c}\mathrm{CO} \\
{\left[\mathrm{mg} \mathrm{m}^{-3}\right]}\end{array}$ & $\begin{array}{l}100(15 \text { min-once per day }) \\
35(1 \mathrm{~h}-\text { once per day }) \\
10,000(8 \mathrm{~h}) \\
7(24 \mathrm{~h}) \text { all from WHO. }\end{array}$ & $\begin{array}{l}10 \text { (max daily } 8 \mathrm{~h} \text { mean) EU } \\
30(1 \mathrm{~h}) \mathrm{WHO} \\
10(8 \mathrm{~h}) \mathrm{WHO}\end{array}$ & {$[13,14]$} \\
\hline $\begin{array}{c}\mathrm{CO}_{2} \\
{[\mathrm{ppm}]}\end{array}$ & $\begin{array}{l}<1000 \text { (hygienically harmless) } \\
\text { 1000-2000 (elevated) } \\
>2000 \text { (hygienically unacceptable) all from AIR. }\end{array}$ & $\begin{array}{l}405 \text { (by climate.gov, accessed on } \\
21 \text { March 2021) }\end{array}$ & [15] \\
\hline $\mathrm{HCHO}\left[\mu \mathrm{g} \mathrm{m}^{-3}\right]$ & $100(30 \mathrm{~min}) \mathrm{WHO}$ & $\mathrm{N} / \mathrm{A}$ & [13] \\
\hline Naphthalene $\left[\mu \mathrm{g} \mathrm{m}^{-3}\right]$ & 10 (annual) WHO & $\mathrm{N} / \mathrm{A}$ & [13] \\
\hline $\begin{array}{c}\mathrm{NO}_{2} \\
{\left[\mu \mathrm{g} \mathrm{m}^{-3}\right]}\end{array}$ & $\begin{array}{l}200(1 \mathrm{~h}) \mathrm{WHO} \\
40 \text { (annual) WHO }\end{array}$ & $\begin{array}{l}200 \text { (1 h) EU/WHO } \\
40 \text { (annual) EU/WHO }\end{array}$ & {$[13,14]$} \\
\hline $\begin{array}{c}\mathrm{O}_{3} \\
{\left[\mu \mathrm{g} \mathrm{m}^{-3}\right]}\end{array}$ & $\mathrm{N} / \mathrm{A}$ & $\begin{array}{l}120 \text { (max daily } 8 \mathrm{~h} \text { mean) EU } \\
100 \text { ( } 8 \mathrm{~h} \text { ) WHO }\end{array}$ & {$[14,16]$} \\
\hline $\begin{array}{c}\text { PAH (benzo[a]pyrene) } \\
{\left[\mathrm{gg} \mathrm{m}^{-3}\right]}\end{array}$ & $\begin{array}{l}\text { All indoor exposures relevant to health, } \\
\text { lung cancer with risk of } 8.7 \times 10^{-8} \text { at } 1 \mu \mathrm{g} \mathrm{m}^{-3} \text {. }\end{array}$ & $\begin{array}{l}1 \text { (annual) EU } \\
0.12 \text { (annual) WHO }\end{array}$ & {$[13,14]$} \\
\hline $\begin{array}{c}\mathrm{PM}_{2.5} \\
{\left[\mu \mathrm{g} \mathrm{m}^{-3}\right]}\end{array}$ & $\begin{array}{l}10 \text { (annual) WHO } \\
25(24 \mathrm{~h}) \mathrm{WHO}\end{array}$ & $\begin{array}{l}10 \text { (annual) WHO } \\
25 \text { (24 h) WHO } \\
25 \text { (annual) EU }\end{array}$ & {$[13,14,16]$} \\
\hline $\begin{array}{c}\mathrm{PM}_{10} \\
{\left[\mu \mathrm{g} \mathrm{m}^{-3}\right]}\end{array}$ & $\begin{array}{l}20 \text { (annual) WHO } \\
50(24 \mathrm{~h}) \mathrm{WHO}\end{array}$ & $\begin{array}{l}20 \text { (annual) WHO } \\
50 \text { (24 h) WHO } \\
40 \text { (annual) EU } \\
50(24 \text { h) EU }\end{array}$ & {$[13,14,16]$} \\
\hline $\begin{array}{l}\text { Tetrachloroethylene } \\
\qquad\left[\mathrm{gg} \mathrm{m}^{-3}\right]\end{array}$ & 250 (annual) & $\mathrm{N} / \mathrm{A}$ & [13] \\
\hline $\begin{array}{l}\text { Trichloroethylene } \\
\qquad\left[\mu \mathrm{g} \mathrm{m}^{-3}\right]\end{array}$ & Carcinogenicity with risk of $4.3 \times 10^{-7}$ at $1 \mu \mathrm{g} \mathrm{m}^{-3}$ & $\mathrm{~N} / \mathrm{A}$ & {$[13]$} \\
\hline $\begin{array}{l}\text { TVOCs }^{\mathrm{a}} \\
{\left[\mathrm{mg} \mathrm{m}^{-3}\right]}\end{array}$ & $\begin{array}{l}<0.3 \text { (no hygienic objections) } \\
>0.3-1 \text { (no relevant objections) } \\
>1-3 \text { (some objections) } \\
>3-10 \text { (major objections) } \\
>10-25 \text { (not acceptable) }\end{array}$ & $\mathrm{N} / \mathrm{A}$ & [17] \\
\hline
\end{tabular}

Note: N/A refers to not available; AIR refers to German Committee on Indoor Guide Values, formerly known as "Ad-hoc AG". a Total VOCs, defined by the International Organisation for Standardisation (ISO) 16000-6. 
Rapid developments in low-cost sensors (LCSs) and wireless communication technologies have become much more prominent in in everyday life [9,18-22]. LCSs have the potential to revolutionise insufficient IAQ monitoring systems with the prospect of delivering real-time air pollution data through sensor networks, which complement the established reference measurement methods defined in the EU Air Quality Directives (e.g., 2008/50/EC). Although there is no officially agreed definition of the term "low-cost" [23], "low-cost" has been identified by the United States Environmental Protection Agency (US EPA) as devices costing less than $\$ 2500$ (USD); this is the limit often defining capital investment limits by LCS users [24]. Due to the importance of this technology in the future development of smart homes, the EU has invested millions of Euros on a number of sensor-based projects such as EuNetAir, IAQSense, and SENSIndoor and the similar investments can be seen elsewhere (e.g., USA, Australia). Smart homes can enable an adaptable living environment, e.g., in managing IAQ, to promote comfort and convenience to the occupants. The outcomes of these projects could contribute in the development of novel sensor systems, real-time air pollution monitors, air quality models, and standardised methods [25-28]. These projects have encouraged research institutions and businesses to take a greater interest in the advancements of sensing technology in IAQ-related research inside smart homes. In the subsequent text, we have interchangeably used the terms "sensor", "sensor kit" and "LCS".

Deploying a network of air pollution LCSs with the support of advanced communication technologies is sufficient to provide accurate information in understanding the spatio-temporal distribution of indoor air pollutants and assessment of personal exposures in smart homes. However, among the limited studies focused on LCS applications in indoor environments, studies mainly focused on general applications, benefits/challenges, future demands/directions of LCSs for specific indoor applications [11,23,29,30]. Moreover, their focus on data analysis was limited to changes in concentrations and no prediction or precautionary actions against the possible events were incorporated [31,32]. Although these studies presented promising results, their scope was not to consider the needs of smart homes. Given the scattered information and research gaps in the existing body of literature, this review aims to fill this knowledge gap by summarising the relevant knowledge in different research disciplines, synthesising the emerging themes and providing unique insights for making homes smart with respect to air quality. In particular, the specific objectives of this review are to (i) provide a comprehensive summary of common indoor air pollutants and pros/cons of LCSs manufactured for indoor applications, (ii) review and summarise the optimal deployment strategies of LCSs within a domestic context, (iii) discuss pre-/post-processing protocols to conduct reliable measurements, carry out data management and data processing, and generate useful information for occupants from the large datasets obtained by networked structures, (iv) evaluate the effectiveness of predictive modelling tools to obtain best-fit approaches with an adequate spatial resolution for estimating exposure to indoor air pollution using LCSs.

\section{Scope and Outline}

The scope of this review is limited to advanced automation technologies, including sensor selection, deployment strategies, data processing, and development of predictive models, which brings healthier indoor environments via monitoring and control of IAQ through the use of networked LCSs. Therefore, a discussion on how to improve IAQ for healthier environments, as well as considerations of ventilation settings, optimal selection of filtration units or air purifying systems are excluded from the scope of this study.

We searched peer-reviewed research articles focusing on the main keywords in various scientific electronic resources, such as Web of Science, ScienceDirect, Wiley, Springer, PubMed and those known to authors. The terminologies used in the search were either one or a combination of "smart homes", "home automation", "low-cost sensors", "affordable pollution sensing", "sensor deployment strategies", "data collection", "data assimilation", "data processing", "machine learning", "data modelling", "predictive modelling", "indoor 
air pollution" and "indoor air quality". Documents published in the technical reports or the following websites, WHO, UK Department for Environment, Food and Rural Affairs (DEFRA), European Commission, US EPA organisations, and different sensor manufacturers were also reviewed for their contents. Since the topic is broad and explored by private companies/organisations as well, studies/reports outside the aforementioned databases were reviewed as a part of a grey literature review using similar keywords through the Google search engine. Studies that did not have one of the quoted terms or explicitly focused on outdoor/ambient environments were discarded from the analysis. The resulting documents were manually screened and collected information fitting to the context was selected for discussion. The search was limited to English-based articles/reports.

As summarised in Figure 1, the article starts with a brief review of the common indoor air pollutants for developing the background context for the topic areas covered (Section 3), followed by the state-of-the-art air pollution sensing technologies for indoor environments by considering their performance and drawbacks (Section 4). The subsequent section (Section 5) explores optimal deployment strategies to better capture spatio-temporal distribution of indoor air pollutants and addresses how these strategies could be built to improve the accuracy and reliability of data. Section 6 summarises pre- and post-processing methods and tools in dealing with LCSs data. Section 7 describes suitability of advanced IAQ predictive models for indoor settings. Finally, a summary of topic areas covered, conclusions and future remarks are presented in Section 8.

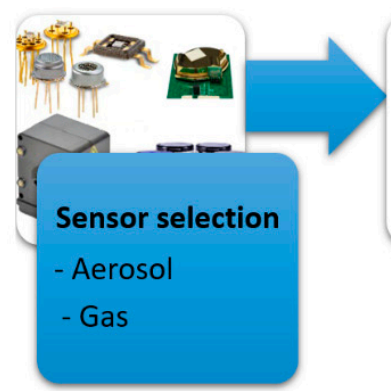

$\underline{\text { Sensors }}$

- Optical

- Metal oxides

- Electrochemical

-Photoionisation detectors

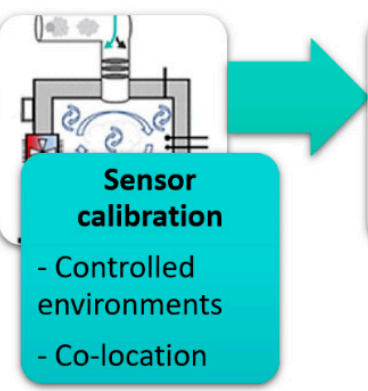

- Pre-evaluation in Envilution ${ }^{\otimes}$ Chamber - Co-location with reference instruments

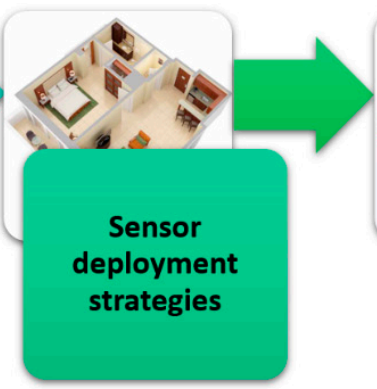

- Including data acquisition, storage in cloud, and communication

- Routine performance evaluation of deployed sensors is required for long term monitoring

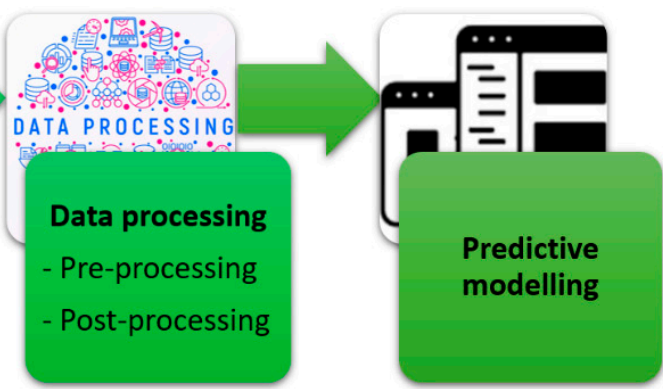

Converting raw data from sensor network to processed data after sufficient $Q A / Q C$

Processed and predicted data for visualisation to home occupants

Figure 1. Essential steps toward a successful implementation of smart indoor sensor network in achieving appreciable indoor air quality (IAQ) and health benefits to home occupants.

\section{Common Indoor Air Pollutants and Their Sources}

IAQ is affected by diverse ranges of indoor sources as well as infiltration of outdoor air pollutants. Each source could impact the overall IAQ, depending on their intensity and the operational time (see Table 1). The most common indoor air pollutants arising from indoor occupants, activities and/or materials are $\mathrm{CO}_{2}, \mathrm{CO}$, VOCs, and PM in different aerodynamic size fractions, including $\mathrm{PM} \leq 2.5 \mu \mathrm{m}\left(\mathrm{PM}_{2.5}\right)$ and $\leq 10 \mu \mathrm{m}\left(\mathrm{PM}_{10}\right)$. Although there can be other pollutants, such as polycyclic aromatic hydrocarbons (PAHs; specifically, benzo[a]pyrene), nitrogen oxides $\left(\mathrm{NOx}=\mathrm{NO}+\mathrm{NO}_{2}\right)$, ozone $\left(\mathrm{O}_{3}\right)$, sulphur dioxide $\left(\mathrm{SO}_{2}\right)$, formaldehyde $(\mathrm{HCHO})$, radon and persistent organic pollutants (POPs), the presence of all of these components in one place is unlikely. In addition, under-controlled thermal comfort parameters, such as temperature, air velocity, relative humidity $(\mathrm{RH})$, noise and lighting levels are other parameters that make the living environment pleasant for the occupants. Hence, a flow of clean air throughout a building environment is necessary to minimise the risk of accumulation of indoor air pollutants. 


\section{Sensor Technology}

Assessing the existing IAQ and unexpected changes in its level through continuous measurement is necessary to know the status of IAQ and its effects on the occupants health. Sensing IAQ with the help of LCSs could be served as the core of smart homes and counted as one of the major components to maintain high-quality living standards. The desirable sensors in smart homes should: (i) be sensitive and selective to target pollutants for reliable sensing relevant to indoor environments that pose health risks to occupants; (ii) be durable with optimal performance over a long-term of deployment; (iii) be small in size, maintenance-free with low-power consumption; (iv) be adopted in complex sensor networks; and (v) work quietly with minimum operating noise [11,12,33-37]. These features enable air pollution sensors to be deployed with relative ease to locations where understanding air quality level could have a huge impact on human health. However, LCSs come with challenges, which may reduce user trust, accuracy and interpretability of recorded data $[12,38]$. If their quality remained unchanged under realistic conditions, they could become a game-changer in various IAQ measurements [39].

\subsection{Electrochemical Sensors}

Electrochemical technology is one of the oldest and perhaps widely used technologies for concentration measurements of gaseous pollutants using either potentiometric (measuring a difference of potentials) or amperometric (measuring current of a redox reaction) principles. Fundamentally, electrochemical sensors (ECs) require at least two electrodes (reference and counter electrodes) for operation, which operate based on a chemical reaction between a gaseous pollutant in the air and an electrode in an electrolyte. The sensors are coated with a catalyst that provides a high surface area, which promotes reactions [34]. The recent ECs contain a cell with three electrodes including, measuring, reference and counter electrodes, which host reduction/oxidation of chosen gases. In this technology, the sample gas diffuses through the sensor's membranes towards the measuring electrode, which results in an electron transfer (produce an internal current). Recently, some sensor manufacturers (e.g., those of AlphaSense and Membrapor, Wallisellen, Switzerland) have upgraded ECs by adding the fourth electrode to monitor physical changes and measure drift [40].

ECs have a comparatively low-cost, high sensitivity/low cross-sensitivity, low detection limit ( s sub-ppm), reasonable response time, and less power-intensive $(\mu \mathrm{W})$ characteristics compared to traditional monitors [34]. Additionally, stability with acceptable drift values (between $2 \%$ and $15 \%$ per year) have been reported for the commercial ECs (e.g., Nemoto and SGX Sensortech) [40]. However, they are more complicated, vulnerable to poisoning, large in size, of shorter life span ( 1-3 years), and more expensive than that of metal oxide semiconductor (MOx) gas sensors (see Section 4.2). As listed in Table 2, ECs have shown interference with the change in meteorology (e.g., air temperature), which is in the first-order impact on an electric output signal of gas concentration (ppb level) and second-order error on gas sensitivity. Low temperatures decrease the speed of reaction in electrochemical cells, which reduce the applicability to operate under cold environments $\left(<10^{\circ} \mathrm{C}\right)$. However, there is a solution to overcome the effects of temperature on background currents (zero currents) that would make a significant impact on measurements at low concentration levels [41].

\subsection{Metal Oxide Semiconductor (MOx) Sensors}

In MOx sensors, gaseous air pollutants react with the sensor surface and change it's electrical (resistance or conductivity) properties [44,45]. Measuring the changes in electrical properties represent the concentration of the target pollutant in the air. Because of advances in fabrication methods and the simplicity of semiconductor sensor devices, MOx gas sensors are moderately low-priced compared to other technologies (cheaper than ECs). MOx sensors are robust, lightweight/long-lasting, sensitive to low-concentration gases (as low as ppb level), and less power intensive (less than $1 \mathrm{~W}$ ) but higher than PIDs (photoionisation detectors; see Section 4.3) [46-48]. 
Simple and fast production processes on a large scale as well as simply controllable processes make MOx gas sensors a desirable technology for air quality monitoring. MOx gas sensors have been reported to be sensitive to a variety of air pollutants [48], with responses changing with the concentration of gaseous pollutants and device operating temperature [46]. MOx gas sensors have been implemented to measure/monitor trace amounts of gaseous pollutants, such as $\mathrm{CO}, \mathrm{CO}_{2}, \mathrm{O}_{3}$, total VOCs, Ammonia $\left(\mathrm{NH}_{3}\right)$ and $\mathrm{NO}_{\mathbf{x}}[46,49]$. However, non-linear output signals, cross-sensitivity to other gases (especially to changes in environmental conditions and other VOC substances in complex mixtures), poisoned by certain or high doses of target gases (e.g., high concentration of certain organic compounds and gaseous sulphur-containing substances) have been discussed in the literature $[12,48,50,51]$.

Table 2. The current available air quality LCSs characteristics, advantages and disadvantages [39,40,42,43]. Examples of different deployed environmental and air pollution LCSs suggested for indoor environments are presented at the end of table.

\begin{tabular}{|c|c|c|}
\hline $\begin{array}{c}\text { Sensor } \\
\text { Technology }\end{array}$ & Known for & Summary of Pros and Cons \\
\hline Electrochemical & $\begin{array}{c}\mathrm{CO}, \mathrm{CO}_{2}, \mathrm{H}_{2}, \mathrm{O}_{3} \\
\mathrm{NH}_{3} \\
\mathrm{NO}, \mathrm{NO}_{2}, \mathrm{NOx} \\
\mathrm{CH}_{4} \\
\mathrm{C}_{3} \mathrm{H}_{8} \text { and VOCs }{ }^{4}\end{array}$ & $\begin{array}{l}\sqrt{ } \text { Good sensitivity, from } \mathrm{mg} \mathrm{m}^{-3} \text { (potentiometric) to } \mu \mathrm{g} \mathrm{m}^{-3} \text { (amperometric). } \\
\sqrt{ } \text { Fast response time }(30-200 \mathrm{~s}) .^{2} \\
\sqrt{ } \text { Small in size }(20 \mathrm{~mm}) \text { and low power consumption }(\mu \mathrm{W}) \text {. } \\
\sqrt{ } \text { Long-term stability with acceptable drift values (between } 2 \% \text { and } 15 \% \text { per year) } \\
\text { reported for the commercial ECs. } \\
\times \text { Large in size, complicated, vulnerable to poisoning, and shorter life span }(\sim 1-3 \text { years). } \\
\times \text { Highly sensitive to change in meteorology (temperature and } \mathrm{RH} \text { variations) } \\
\text { depending on electrolyte. }{ }^{3} \\
\times \text { Show cross-reactivity with similar molecule types. } \\
\times \text { More expensive than } \mathrm{MOx} \text { gas sensors. } \\
\sqrt{ } \text { Good sensitivity, from } \mathrm{mg} \mathrm{m}^{-3} \text { to } \mu \mathrm{g} \mathrm{m}^{-3} \text { (ppb level) and relatively long lifetime }(>5 \\
\text { years). } \\
\sqrt{ } \text { Small in size (few millimetres) and long-lasting } / \text { light weight (few grams). } \\
\sqrt{ } \text { Least power intensive }(<1 \mathrm{~W})-\text { but higher than PIDs. } \\
\times \text { Results are affected by temperature and RH variations. } \\
\times \text { Long response time }(>30 \mathrm{~s} ; \text { some cases } 5-50 \text { min), long stabilisation period before } \\
\text { measurements }(\sim 24 \mathrm{~h}), \text { and longer-term performance drift. } \\
\times \text { Poor recovery to achieve initial status under a change in experimental condition or } \\
\text { exposure to a high concentration of target gases. } \\
\times \text { Output depends on the history of past inputs. } \\
\times \text { Instability over time. } 5\end{array}$ \\
\hline PID & VOCs $^{1}$ & $\begin{array}{l}\sqrt{ } \text { Small in size with moderate price (approximately } 400 € \text { for a sensor to } \sim 5000 € \text { for a } \\
\text { handheld device). } \\
\sqrt{ } \text { Good sensitivity, down to } \mathrm{mg} \mathrm{m}^{-3} \text {, some down to } \mu \mathrm{g} \mathrm{m}^{-3} \text {. } \\
\sqrt{ } \text { Limited temperature dependence and } \mathrm{RH} \text { effects. } \\
\sqrt{ } \text { Very fast (a few) response time. } \\
\times \text { Not selective: reacts to all VOC that can be ionised by the UV lamp. Proper } \\
\text { calibration and maintenance may be needed. } \\
\times \text { Significant signal drift. }\end{array}$ \\
\hline $\begin{array}{l}\text { Optical particle } \\
\text { counter }\end{array}$ & PMs & $\begin{array}{l}\sqrt{ } \text { Fast response time (in a second). } \\
\sqrt{ } \text { Sensitivity in the range of } 1 \mu \mathrm{g} \mathrm{m}{ }^{-3} \text {. } \\
\sqrt{ } \text { Able to identify the size of the particle in the size of } \mathrm{PM}_{10} \text { and } \mathrm{PM}_{2.5} . \\
\times \text { Conversion from PM counts to PM mass with the theoretical model. } \\
\times \text { The measured signal depends on a variety of parameters such as particle shape, } \\
\text { colour and density, RH, refractive index, etc. } \\
\times \text { Unable to detect ultrafine particles. } 6\end{array}$ \\
\hline
\end{tabular}


Table 2. Cont.

\begin{tabular}{cll}
\hline $\begin{array}{c}\text { Sensor } \\
\text { Technology }\end{array}$ & Known for & \multicolumn{1}{c}{ Summary of Pros and Cons } \\
\hline & & $\sqrt{ }$ Good sensitivity for $\mathrm{CO}_{2}(350-2000 \mathrm{ppm})$. \\
& & $\sqrt{ }$ Selectivity is good through characteristic $\mathrm{CO}_{2} \mathrm{IR}$ spectra. \\
Optical & $\mathrm{CO}$ and $\mathrm{CO}_{2}$ & $\sqrt{ }$ Response time 20-120 s. \\
& $\sqrt{ }$ Limited drift over time of the sensor calibration. \\
& $\times$ Need for correction for the effects of temperature, $\mathrm{RH}$ and pressure. \\
\hline
\end{tabular}

${ }^{1}$ Photoionisation detectors (PIDs) demonstrate a better sensitivity than electrochemical cells for volatile organic compounds (VOCs) (range from $100 \mathrm{ppb}$ and $20 \mathrm{ppm}$ ).

2 Depend on the air temperature [40].

3 The interference caused by temperature influence can be compensated.

${ }^{4} \mathrm{MOx}$ should not be used to measure low concentrations of VOCs in the presence of high concentrations of $\mathrm{NO}, \mathrm{NO}_{2}$ or $\mathrm{CO}$. $\mathrm{MOx}$ sensors are suitable when sensing VOCs, which are not detected by PIDs (e.g., many chlorofluorocarbons (CFCs)) [40].

${ }^{5}$ An empirical relation for drift or stability corrections have been suggested $[40,44]$.

${ }^{6}$ No LCS is available that could detect ultrafine particles ( $<100 \mathrm{~nm}$ in diameter), because the optical systems are unable to detect $<300 \mathrm{~nm}$ particles [42].

Note 1: Near real-time monitoring in indoor environments is required to capture the immediate incidents and to adopt precautionary and corrective measures, but not all the sensors discussed above are fast/immediate responsive enough to concentration changes. Currently, a reasonable average time among the deployed sensors is 30-s and/or 1-min averaging timestamp as per published studies in the literature. Besides, a balance should be maintained between sampling frequency and power source. Note 2: There are LCSs for other pollutants, such as Radon, $\mathrm{NO}, \mathrm{H}_{2} \mathrm{~S}$, and $\mathrm{SO}_{2}$ which are not listed in here.

Note 3: Here are some sensors that have been used in IAQ studies:

- $\quad$ Temperature/relative humidity (RH)/pressure: AM2302 (Adafruit, New York, USA), BME280/680 (Bosch GmbH), HDC1080 (Texas Instruments Co., USA), SHT-31 (Sensirion, Switzerland).

- $\quad$ Sound/noise: ICS-434342 (Invensense) and Adafruit \#1063.

- $\quad$ Light: BH1721FVC (ROHM Semiconductor) and TSL2561 (Texas Advanced Optoelectronic Solutions).

- $\quad$ Particulate matter $\left(\mathrm{PM}_{1 / 2.5 / 10}\right)$ : some of the PM sensors such as the GP2Y1010AU0F (Sharp Corporation, Osaka, Japan), DSM501A (Samyoung S\&C, Seongnam-si, South Korea), PPD42NS (Shinyei Technology, Japan), and PPD60PV (Shinyei Technology Co., Kobe, Japan) cannot distinguish between particle sizes and report single mass concentration of particles (sizes $>\sim 0.3 \mu \mathrm{m}$ ) in air. However, other sensor manufacturers such as HPMA115S0 (Honeywell Sensing Inc., Charlotte, NC, USA), OPC-N2/3 (Alphasense, Braintree, UK), Plantower PMS series, such as 5003 and 7003 (Beijing Plantower Co., Ltd., Beijing, China), ZH03A (Zhengzhou Winsen Electronics Technology Co., Ltd., Zhengzhou, China), SDL301/607 and SDS011/018/021 (Nova Fitness Co., Ltd., Jinan, China) rely on different size bins. There are other sensors such as household air pollution exposure (HAPEx) and TZOA-r for PM measurements.

- $\quad$ CO: CO-A44/-B41 (EC; Alphasense, UK), 4-CO-500 (EC; Euro-Gas Management Services LTD., Brixham, UK), 110-102 (EC; SPEC Sensors, LLC), MQ-7 (MOx; Zhengzhou Winsen Electronics Technology Co., Ltd., China), MICS-5525 (MOx; SGX-Sensotech, Corcelles, Switzerland), TGS-5042 (EC; Arlington Heights, IL) and EL-USB-C. For both MOx and EC CO sensors, poor performance $\left(\mathrm{R}^{2} \approx 0.1\right)$ was observed in long-term deployment (4.5 months). Hence, routine in-field calibration should be accounted to avoid aging [40].

- $\quad \mathrm{CO}_{2}$ : ELT $\mathrm{S} 300$ (NDIR (nondispersive infrared); ELT Sensor Corp., Bucheon-si, Korea), TGS 4161-type (NDIR; FIGARO USA, Inc., Arlington Heights, IL, USA), INE20-CO2P-NCVSP, SST CO2S-A (NDIR; SST Technologies), and T6713 (NDIR; Amphenol Advanced Sensors, St Marys, PA, USA).

- $\quad$ TVOCs: BME680 (MOx; Bosch GmbH), CCS811 (MOx; ScioSense, Eindhoven, The Netherlands), and MiCS-VZ-89TE (MOx; Amphenol Advanced Sensors, USA).

- $\quad \mathrm{NO}_{2}$ : OX-B431, NO2-A43F/-B43F (EC; Alphasense, $\mathrm{UK}, \mathrm{NO}_{2}$ sensor with $\mathrm{O}_{3}$ filter to minimise the $\mathrm{O}_{3}$ interference), $\mathrm{NO} 2 \_3 \mathrm{E} 50$ (EC; Citytech, UK), and MICS-2710/4514 (MOx; SGX-Sensotech). Excellent performance $\left(\mathrm{R}^{2} \approx 1\right)$ under laboratory conditions, while poor performance under field conditions was achieved that highlights the necessity for careful performance evaluation.

- $\quad \mathrm{O}_{3}$ : MICS-2610/2611 (EC; SGX Sensortech, Switzerland), MQ131 (MOx; ETC), OX-A431/-B431/-B421/O3B4 (EC; Alphasense, UK), and O3_3E1F (EC; Life Safety Germany GmbH, München, Germany). Both EC and MOx sensors performed well under controlled laboratory conditions $\left(R^{2}>0.9\right)$; however, their performance gets decayed under field conditions $\left(R^{2}=0.01-0.94\right)$. Temperature, $\mathrm{RH}$ and cross-sensitivity to $\mathrm{CO}, \mathrm{CO}_{2}, \mathrm{NO}, \mathrm{NO}_{2}, \mathrm{SO}_{2}$, and $\mathrm{NH}_{3}$ have been reported as drawbacks that affect the outputs [23].

\subsection{Photoionisation Detectors (PIDs)}

The PID is another type of LCS, which uses high-energy photons (ultraviolet (UV) light) for ionisation of gaseous molecules [40]. The main principle is that the gas between 
the electrodes is ionized by UV light (in the energy scale of $10 \mathrm{eV}$ ) to produce charged ions. The resulting ions are proportional to the output signals as well as pollutant concentrations in the detector. Due to high sensitivity, PIDs are extensively used for the detection of VOCs, because each VOC component has its own ionisation potential (IP). IP range varies from easy to ionise substances $(\sim 7 \mathrm{eV})$ to extremely difficult to ionise substances $(\sim 12-16 \mathrm{eV})$. For example, PIDs effectively detect most hazardous gases, including VOCs (e.g., benzene $=9.25$; hexane $=10.13$; toluene $=8.82$; and xylene $=8.56 \mathrm{eV})$ due to their low IPs, and offer a range of benefits, such as fast response, small size, ease of use/maintenance, and ability to detect low concentrations. However, PIDs cannot detect air constituents $\left(\mathrm{O}_{2}\right.$ and $\mathrm{N}_{2}$ ), $\mathrm{CO}_{2}, \mathrm{CO}, \mathrm{SO}_{2}, \mathrm{CH}_{4}$, and $\mathrm{O}_{3}$ due to their high IPs.

\subsection{Optical Sensors}

Optical sensors, also called light scattering sensors, are used for detection of PMs. Light-scattering PM sensors measure the optical properties of the particles as an ensemble, which offers fast and real-time responses, minimal drift and greatly reduces the cost and size of the sensors [52-55]. In addition to small size, low-energy consumption (less power supply voltage $\sim 5 \mathrm{~V}$ ) and ability to generate high-frequency output data during operations make optical sensors a good candidate in various applications $[56,57]$. Furthermore, variations in $\mathrm{PM}_{2.5}$ concentration measurement under low-concentrations $\left(20-30 \mu \mathrm{g} \mathrm{m}^{-3}\right)$ among different optical PM sensors against reference instruments could be a major drawback of sensors of this type. This is because the amount of scattered light is reliant on size, shape, density, and refractive index of particles [58]. Despite all these limitations, reliable functioning of optical PM sensors in indoor environments with small spatial scale was reported [59].

\subsection{Sensor Selection}

Putting multiple sensors together onto boards, calibrating and reshaping them as commercial products for indoor (or outdoor) applications has been a common practice. Such sensor-based products are becoming increasingly available, while the information around lifetime and maintenance are not clearly available. Table 2 (sensors) and Table 3 (commercial sensor-based products) summarise the specification of technologies in the market, whose performances have been evaluated by at least one indoor study. Moreover, the manufacturer's specifications obtained from technical datasheets, such as type of pollutant, technology, measuring range, reported sensor lifetime, sampling mechanism, sampling interval, environmental operating range, and connectivity have been summarised in Table 3.

Studies showed that the sensor correlations against the research-grade instruments could vary before and/or after deployment even for identical sensors under identical conditions [60-63]. Furthermore, environmental conditions (temperature and $\mathrm{RH}$ ) and cross-sensitivities of certain pollutants (e.g., $\mathrm{NO}_{2}$ gas on $\mathrm{O}_{3}$ sensors, $\mathrm{NO}$ gas on $\mathrm{NO}_{2}$ sensors, and hydrogen molecule on $\mathrm{CO}$ sensors) on sensor readings have been imperfectly addressed $[34,38,64-66]$. In other words, due to the lack of regulatory bodies, questions are raised about their reported values, reproducibility and comparability. However, significant progress has been made in this direction in the recent past. For example, the Air Quality Sensor Performance Evaluation Centre (AQ-SPEC) operated by South Coast Air Quality Management District (SCAQMD) [67,68], the US EPA, Air Sensor Toolbox [69], and the EU Joint Research Centre (EU JRC) [50,70] programs have been initiated to quantitatively evaluate the performance, stability and quality assurance/control (QA/QC) of sensor-based products. To tackle these issues in a more convenient way by not only considering in-field co-location, field normalisation or field calibration with reference instruments [71-73], recent studies have shown an alternative solution that can be utilised to improve the QA/QC of readings. Affordable laboratory facilities, such as the Envilution ${ }^{\circledR}$ chamber are currently offered by academic and research institutions to calibrate and evaluate the performance of LCSs before and after deployment under controlled environments [73]. Here, a controlled 
environment is defined as a situation where changes in environmental conditions and pollution concentrations, representing indoor environments, for testing LCSs can be simulated (controlled) inside the chamber. Therefore, LCSs performance can be assessed under a combination of indoor variations in environmental parameters and pollution concentrations. In-field co-location would be an alternative QA/QC measure after deployment. Moreover, routine calibration checks after deployment for simple networks along with advanced statistical techniques, e.g., data consistency checks, network correlations, and principal components analysis, in complex networks (Section 7) can boost the performance of this system to maintain long-term satisfactory performance. Such platforms, initiatives and programs offer support to obtain reliable data by the use of appropriate sensors, which could result in improving personal exposure estimates in home environments.

Table 3. Specification of sensor-based product specifications (both single- and multiple-purpose units) reported by manufacturers available in the market that could be used for IAQ and/or personal indoor exposure monitoring systems. The authors highly suggest the buyers to check the up-to-date specifications of the sensors prior to selection and do not endorse any brand or a product.

\begin{tabular}{|c|c|c|c|}
\hline \multicolumn{4}{|c|}{ Single-Purpose Units Designed for IAQ } \\
\hline Sensor name & Pollutant & Technology & Specific Practical Features \\
\hline $\begin{array}{l}\text { Aeroqual S500 } \\
\underline{(\mathrm{OZU})}\end{array}$ & $\begin{array}{l}\text { Can be used with a wide } \\
\text { range of gas sensor } \\
\text { heads (e.g., } \mathrm{CO}, \mathrm{CO}_{2}, \mathrm{O}_{3} \text {, } \\
\left.\text { VOCs, } \mathrm{PM}_{2.5} \text { and } \mathrm{PM}_{10}\right) \text {. }\end{array}$ & $\begin{array}{l}\text { A sensitive MOx that } \\
\text { relies } \\
\text { on the conductance of } \\
\text { heated tungstic oxide } \\
\left(\mathrm{WO}_{3}\right) .\end{array}$ & $\begin{array}{l}\text { Battery: Yes (12Vdc } 2700 \mathrm{~mA} . \mathrm{h}) \\
\text { Sampling mechanism: Air pump } \\
\text { Sampling interval: N/S } \\
\text { Environmental operating conditions }-5 \text { to } 45^{\circ} \mathrm{C} \text {; up } \\
\text { to } 95 \% \text { of } \mathrm{RH} \\
\text { Internal data storage/ wireless communication: } \\
\text { Yes/Yes } \\
\text { Calibration: Zero and span calibration }\end{array}$ \\
\hline
\end{tabular}

\begin{tabular}{|c|c|c|c|}
\hline AirAssure by TSI & $\begin{array}{l}\text { Real-time measurements } \\
\text { of } \mathrm{PM}_{2.5} \text { mass } \\
\text { concentrations. }\end{array}$ & $\begin{array}{l}\text { Enable a light-scattering } \\
\text { photometer that detects } \\
\text { and measures } \mathrm{PM}_{2.5} \\
\text { between } 5 \text { and } 300 \mu \mathrm{g} \\
\mathrm{m}^{-3} \text {. }\end{array}$ & $\begin{array}{l}\text { Power supply: Yes ( } 24 \mathrm{~V}, 5 \mathrm{~W} \max ) \\
\text { Sampling mechanism: Air pump } \\
\text { Sampling interval: N/S } \\
\text { Environmental operating conditions: } 10 \text { to } 30^{\circ} \mathrm{C} \text {; } \\
<65 \% \\
\text { Internal data storage/wireless communication: } \\
\text { No/Yes } \\
\text { Calibration: Pre-calibrated sensor with the National } \\
\text { Institute of Standards and Technology (NIST) } \\
\text { Statement of Conformance }\end{array}$ \\
\hline$\frac{\text { AirBeam2 }}{\text { by HabitatMap }}$ & $\begin{array}{l}\text { Measures } \mathrm{PM}_{1}, \mathrm{PM}_{2.5} \\
\text { and } \mathrm{PM}_{10} \text {, temp. and } \\
\text { RH. }\end{array}$ & $\begin{array}{l}\text { Use a light-scattering } \\
\text { method to measure PMs. } \\
\text { Particle sensor } \\
\text { (Plantower PMS7003); } \\
\text { RH sensor (Honeywell } \\
\text { HIH-5030-001); Temp. } \\
\text { sensor (Microchip } \\
\text { MCP9700T-E/TT) }\end{array}$ & $\begin{array}{l}\text { Battery: Yes (up to } 10 \mathrm{~h} \text { battery life) } \\
\text { Power supply: Yes - micro universal serial bus (USB) } \\
\text { port } \\
\text { Sampling mechanism: Air pump } \\
\text { Sampling interval: N/S } \\
\text { Environmental operating conditions: N/S } \\
\text { Internal data storage/wireless communication: } \\
\text { No/Yes } \\
\text { Calibration: Pre-calibrated by the manufacturer }\end{array}$ \\
\hline $\begin{array}{l}\text { Alphasense OPC } \\
\text { - Particulate Monitor }\end{array}$ & $\begin{array}{l}\text { Measures } \mathrm{PM}_{1}, \mathrm{PM}_{2.5} \\
\text { and } \mathrm{PM}_{10} \cdot \\
\text { Certified with ISO } \\
\text { 9001:2015. }\end{array}$ & $\begin{array}{l}\text { Use laser beams to } \\
\text { detect } \\
\text { particles from } 0.38 \\
\text { micron } \\
\text { to } 17 \text { micron in diameter. }\end{array}$ & $\begin{array}{l}\text { Power supply: No battery, } 175 \mathrm{~mA} \\
\text { Sampling mechanism: Air pump } \\
\text { Sampling interval: Histogram period (1-30 s) } \\
\text { Environmental operating conditions: up to } 50{ }^{\circ} \mathrm{C} \text {; up } \\
\text { to } 95 \% \\
\text { Internal data storage/wireless communication: } \\
\text { Yes/No } \\
\text { Calibration: Pre-calibrated by the manufacturer }\end{array}$ \\
\hline
\end{tabular}


Table 3. Cont.

\begin{tabular}{|c|c|c|c|}
\hline \multicolumn{4}{|c|}{ Single-Purpose Units Designed for IAQ } \\
\hline Sensor name & Pollutant & Technology & Specific Practical Features \\
\hline $\begin{array}{l}\text { AS-LUNG } \\
\text { portable }\end{array}$ & $\begin{array}{l}\text { Real-time measurements } \\
\text { of } \mathrm{PM}_{1}, \mathrm{PM}_{2.5} \text { and } \mathrm{PM}_{10} \\
\text { in } \mu \mathrm{g} \mathrm{m} \mathrm{m}^{-3} \text { as well as } \\
\mathrm{CO}_{2} \text { concentrations. }\end{array}$ & $\begin{array}{l}\text { Use Plantower PMS3003 } \\
\text { laser particle counter } \\
\text { sensors, which come } \\
\text { factory calibrated. }\end{array}$ & $\begin{array}{l}\text { Battery: No (Yes for the station) } \\
\text { Power supply: Yes, DC-5V } \\
\text { Sampling mechanism: Air pump } \\
\text { Sampling interval: N/S } \\
\text { Environmental operating conditions: N/S } \\
\text { Internal data storage/wireless communication: } \\
\text { Yes/Yes } \\
\text { Calibration: Pre-calibrated by the manufacturer }\end{array}$ \\
\hline$\underline{\text { Cair }}$ & $\begin{array}{l}\text { Detect dust particle } \\
\text { concentration of a given } \\
\text { size range in pcs } \mathrm{ft}^{-3} \text {. } \\
\text { It measures VOC in ppm } \\
\text { level, air temp. and RH. }\end{array}$ & $\begin{array}{l}\text { Count particle via laser } \\
\text { beams }\end{array}$ & $\begin{array}{l}\text { Power supply: No battery, yes (USB } 5 \mathrm{~V} \text { ) } \\
\text { Sampling mechanism: Air pump } \\
\text { Sampling interval: } 1 \mathrm{~min} \\
\text { Environmental operating conditions: N/S } \\
\text { Internal data storage/wireless communication: } \\
\text { No/Yes } \\
\text { Calibration: Pre-calibrated by the manufacturer }\end{array}$ \\
\hline$\frac{\text { Cairsense }}{\text { micro-sensors }}$ & $\begin{array}{l}\text { Offers a separate range } \\
\text { of air quality gas sensors, } \\
\text { including } \mathrm{T}, \mathrm{RH}, \mathrm{NO}_{2}, \\
\mathrm{NH}_{3}, \mathrm{CO}, \mathrm{O}_{3}+\mathrm{NO}_{2} \\
\mathrm{NH}_{3}, \mathrm{H}_{2} \mathrm{~S}+\mathrm{CH}_{4} \mathrm{~S}, \\
\mathrm{HCHO}, \mathrm{SO}_{2}, \mathrm{PMs}_{\text {, and }} \\
\text { non-methane VOCs. }\end{array}$ & $\begin{array}{l}\text { See Technical Data of } \\
\text { each sensor kit for } \\
\text { detailed specifications. }\end{array}$ & $\begin{array}{l}\text { Battery: Yes } \\
\text { Power supply: } 5 \text { VDC / } 500 \mathrm{~mA} \\
\text { Sampling mechanism: Air pump } \\
\text { Sampling interval: } 1,15 \text {, and } 60 \mathrm{~min} \\
\text { Environmental operating conditions: up to } 40^{\circ} \mathrm{C} \text {; up } \\
\text { to } 100 \% \\
\text { Internal data storage/wireless communication: } \\
\text { Yes/Yes } \\
\text { Calibration: Pre-calibrated by the manufacturer }\end{array}$ \\
\hline$\underline{\text { Dylos - DC1700-PM }}$ & $\begin{array}{l}\text { Measures both } \mathrm{PM}_{2.5} \\
\text { and } \mathrm{PM}_{10} \text { number }(>0.5 \\
\mu \mathrm{m} \text { and }>2.5 \mu \mathrm{m}) \text { and } \\
\text { mass concentrations } \\
\text { interchangeably. }\end{array}$ & $\begin{array}{l}\text { Use a true laser particle } \\
\text { counter, where laser } \\
\text { beams detect particles } \\
\text { going past by their } \\
\text { reflectivity. }\end{array}$ & $\begin{array}{l}\text { Battery: Yes (up to } 6 \text { h of continuous use) } \\
\text { Power supply: Yes } \\
\text { Sampling mechanism: Air pump } \\
\text { Sampling interval: Minimum for } 1 \text { min } \\
\text { Environmental operating conditions: N/S } \\
\text { Calibration: Pre-calibrated by the manufacturer }\end{array}$ \\
\hline$\underline{\text { Eco Witt WH43 }}$ & $\begin{array}{l}\text { Designed to provide } \\
\text { real-time measurements } \\
\text { of } \mathrm{PM}_{2.5} \text { mass } \\
\text { concentrations. }\end{array}$ & $\begin{array}{l}\text { Use Honeywell HPM } \\
\text { Series Particulate Sensor } \\
\text { to detect/count particles } \\
\text { using light-scattering } \\
\text { between 0-999 } \mathrm{\mu g} \mathrm{m}^{-3} \text {. }\end{array}$ & $\begin{array}{l}\text { Battery: Yes } \\
\text { Power supply: Yes (USB power cable) } \\
\text { Sensor lifetime: } 10 \text { years for the Honeywell HPM } \\
\text { Series } \mathrm{PM}_{2.5} \text { sensor } \\
\text { Sampling mechanism: Air pump } \\
\text { Sampling interval: N/S } \\
\text { Environmental operating conditions: N/S } \\
\text { Internal data storage/wireless communication: } \\
\text { No/Yes } \\
\text { Calibration: Pre-calibrated by the manufacturer }\end{array}$ \\
\hline$\underline{\text { Laser Egg }}$ & $\begin{array}{l}\text { A handheld device that } \\
\text { provides real-time } \\
\text { measurements of } \mathrm{PM}_{2.5} \\
\text { and } \mathrm{PM}_{10} \text {. }\end{array}$ & $\begin{array}{l}\text { Use light-scattering to } \\
\text { measure particles } \\
\text { between } 0.3 \text { and } 10 \\
\text { micron within } 10-100 \\
\text { ms in aerodynamic } \\
\text { diameter. }\end{array}$ & $\begin{array}{l}\text { Battery: Yes } \\
\text { Battery Life: } 8 \mathrm{~h} \\
\text { Power supply: DC } 5 \mathrm{~V} \text { (USB charging cable) } \\
\text { Sampling mechanism: Air pump } \\
\text { Sampling interval: N/S } \\
\text { Environmental operating conditions: N/S } \\
\text { Internal data storage/wireless communication: } \\
\text { No/Yes } \\
\text { Calibration: Pre-calibrated by the manufacturer }\end{array}$ \\
\hline
\end{tabular}


Table 3. Cont.

\begin{tabular}{|c|c|c|c|}
\hline \multicolumn{4}{|c|}{ Single-Purpose Units Designed for IAQ } \\
\hline Sensor name & Pollutant & Technology & Specific Practical Features \\
\hline$\underline{\text { Micro Aeth }}$ & $\begin{array}{l}\text { Model AE51 } \\
\text { aethalometer, BC aerosol } \\
\text { monitor that measures } \\
0-1 \mathrm{mg} \mathrm{BC} \mathrm{m}^{-3}\end{array}$ & $\begin{array}{l}\text { Measures the rate of } \\
\text { change in absorption of } \\
\text { transmitted light due to } \\
\text { a continuous collection } \\
\text { of aerosol deposit on T60 } \\
\text { (Teflon coated glass } \\
\text { filter). }\end{array}$ & $\begin{array}{l}\text { Battery: Yes } \\
\text { Power supply: Yes (5 V DC/0.5 A) } \\
\text { Sampling mechanism: Internal pump up to } 200 \mathrm{~mL} \\
\text { min }^{-1} \\
\text { Sampling interval: } 1,10,30,60 \text {, or } 300 \mathrm{~s} \\
\text { Environmental operating conditions: } 0 \text { to } 40^{\circ} \mathrm{C} \\
\text { Internal data storage/wireless communication: } \\
\text { Yes/Yes } \\
\text { Calibration: Pre-calibrated by the manufacturer }\end{array}$ \\
\hline MicroPEM by RTI & $\begin{array}{l}\text { A portable sensing } \\
\text { device that measures } \\
\mathrm{PM}_{2.5} \text { and } \mathrm{PM}_{10} .\end{array}$ & $\begin{array}{l}\text { It combines real-time } \\
\text { nephelometry and } \\
\text { integrated referee filter } \\
\text { PM measurements. The } \\
\text { device carries an } \\
\text { impactor and a } \\
\text { light-scattering particle } \\
\text { detector. }\end{array}$ & $\begin{array}{l}\text { Battery: Yes (up to } 40 \mathrm{~h} \text { of continuous operation) } \\
\text { Power supply: Yes ( } 120 \mathrm{~V} \text { AC } / 60 \mathrm{~Hz} \text { AC adapter to } \\
\text { USB) } \\
\text { Sampling mechanism: Pump ( } 500 \mathrm{~mL} \mathrm{~min}{ }^{-1} \text { ) } \\
\text { Sampling interval: } 10 \mathrm{~s} \\
\text { Environmental operating conditions: N/S } \\
\text { Internal data storage/wireless communication: } \\
\text { Yes/Yes } \\
\text { Calibration: Pre-calibrated by the manufacturer }\end{array}$ \\
\hline$\underline{\text { Naneos - Partector }}$ & $\begin{array}{l}\text { A portable, } \\
\text { battery-powered } \\
\text { instrument that } \\
\text { measures the lung } \\
\text { deposited surface area } \\
\text { (LDSA) of nanoparticles. }\end{array}$ & $\begin{array}{l}\text { Measures nanoparticle } \\
\text { surface area based on a } \\
\text { non-contact electrical } \\
\text { detection principle. }\end{array}$ & $\begin{array}{l}\text { Battery: Internal rechargeable Li:Ion battery }(15 \mathrm{~h}) \\
\text { Power supply: USB charger (to either charge or run } \\
\text { indefinitely) } \\
\text { Sampling mechanism: Air pump }\left(0.5 \mathrm{~L} \mathrm{~min}^{-1}\right) \\
\text { Sampling interval: N/S } \\
\text { Environmental operating conditions: N/S } \\
\text { Internal data storage/wireless communication: } \\
\text { Yes/No } \\
\text { Calibration: Pre-calibrated by the manufacturer }\end{array}$ \\
\hline $\begin{array}{l}\text { POM * by } 2 \mathrm{~B} \\
\text { Technologies }\end{array}$ & $\begin{array}{l}\text { Personal Ozone Monitor } \\
\text { (POM) } 4 \mathrm{ppb}-10 \mathrm{ppm} \text {, } \\
\text { Resolution } 0.1 \mathrm{ppb}\end{array}$ & $\begin{array}{l}\text { Absorption of ultraviolet } \\
\text { light } \\
\text { at } 254 \mathrm{~nm} \\
\text { Baseline drift }<2 \mathrm{ppb} \text { per } \\
\text { day, } \\
<5 \mathrm{ppb} \text { per year } \\
\text { Sensitivity drift }<1 \% \text { per } \\
\text { day, } \\
<3 \% \text { per year }\end{array}$ & $\begin{array}{l}\text { Battery lifetime: } 5-8 \mathrm{~h} \\
\text { Power supply: Yes } \\
\text { Sampling mechanism: Air pump }\left(0.75 \mathrm{~L} \mathrm{~min}{ }^{-1}\right) \\
\text { Sampling interval: } 10 \mathrm{~s}, 0.1 \mathrm{~Hz} \text { (Fast mode: } 2 \mathrm{~s}, 0.5 \\
\mathrm{~Hz} \text { ) } \\
\text { Environmental operating conditions: up to } 50^{\circ} \mathrm{C} \\
\text { Internal data storage/wireless communication: } \\
\text { Yes/Yes } \\
\text { Calibration: Pre-calibrated by the manufacturer }\end{array}$ \\
\hline $\begin{array}{l}\text { PurpleAir } \\
\text { PA-II } \\
\text { (IAQ and OAQ) }\end{array}$ & $\begin{array}{l}\text { An OPC, which } \\
\text { measures } \mathrm{PM}_{1}, \mathrm{PM}_{2.5} \\
\text { and } \mathrm{PM}_{10} \text { mass }\end{array}$ & $\begin{array}{l}\text { Use Plantower PMS5003 } \\
\text { laser particle counter } \\
(\text { maximum range } \geq 1000 \\
\mu \mathrm{g} \mathrm{m}^{-3} \text { ), where laser } \\
\text { beams detect particles } \\
\text { going past by their } \\
\text { reflectivity. }\end{array}$ & $\begin{array}{l}\text { Power supply: } 5 \text { V DC, } 3 \text { A } \\
\text { Sampling mechanism: Air pump } \\
\text { Sampling interval: N/S } \\
\text { Environmental operating conditions: N/S } \\
\text { Internal data storage/wireless communication: } \\
\text { No/Yes } \\
\text { Calibration: Pre-calibrated by the manufacturer }\end{array}$ \\
\hline$\frac{\text { PurpleAir }}{\text { PA-I-Indoor }}$ & $\begin{array}{l}\text { concentrations from the } \\
\text { counts. }\end{array}$ & $\begin{array}{l}\text { Use PMS1003 laser } \\
\text { particle counters } \\
\text { (maximum range } \geq 1000 \\
\mu \mathrm{g} \mathrm{m}^{-3} \text { ) where laser } \\
\text { beams detect particles } \\
\text { going past by their } \\
\text { reflectivity. }\end{array}$ & $\begin{array}{l}\text { Power supply: } 5 \text { V DC, } 3 \text { A } \\
\text { Sampling mechanism: Air pump } \\
\text { Sampling interval: N/S } \\
\text { Environmental operating conditions: up to } 60^{\circ} \mathrm{C} \text {; up } \\
\text { to } 99 \% \\
\text { Internal data storage/wireless communication: } \\
\text { No/Yes } \\
\text { Calibration: Pre-calibrated by the manufacturer }\end{array}$ \\
\hline
\end{tabular}


Table 3. Cont.

\begin{tabular}{|c|c|c|c|}
\hline \multicolumn{4}{|c|}{ Single-Purpose Units Designed for IAQ } \\
\hline Sensor name & Pollutant & Technology & Specific Practical Features \\
\hline$\frac{\text { Samyoung S\&C }}{\text { SY-DS-DK3 }}$ & $\begin{array}{l}\text { Designed to provide } \\
\text { real-time measurements } \\
\text { of } \mathrm{PM}_{2.5} \text { mass } \\
\text { concentrations. }\end{array}$ & $\begin{array}{l}\text { The } \mathrm{PM}_{2.5} \text { sensor } \\
\text { provides mass } \\
\text { concentration over } 0.3 \\
\mu \mathrm{m} \text { sized particles } \\
\text { through Samyoung } \\
\text { S\&C's proprietary } \\
\text { optical structure with } \\
\text { Infrared emitting diode. }\end{array}$ & $\begin{array}{l}\text { Power supply: Yes } \\
\text { Sensor lifetime: min. } 5 \text { years } \\
\text { Sampling mechanism: Air pump } \\
\text { Sampling interval: } 2 \mathrm{~s} \\
\text { Environmental operating conditions: up to } 65^{\circ} \mathrm{C} \text {; up } \\
\text { to } 95 \% \\
\text { Internal data storage/wireless communication: N/S } \\
\text { Calibration: Pre-calibrated by the manufacturer }\end{array}$ \\
\hline$\frac{\text { Sensirion - SPS30 }}{\text { Eval Kit }}$ & $\begin{array}{l}\text { Real-time PMs } \\
\text { mass/number } \\
\text { concentrations. } \\
\text { Mass concentration: } \\
\mathrm{PM}_{1.0}, \mathrm{PM}_{2.5}, \mathrm{PM}_{4} \text { and } \\
\mathrm{PM}_{10} \\
\mathrm{Number} \text { concentration: } \\
\mathrm{PM}_{0.5}, \mathrm{PM}_{1.0}, \mathrm{PM}_{2.5} \\
\mathrm{PM}_{4} \text { and } \mathrm{PM}_{10}\end{array}$ & $\begin{array}{l}\text { Based on laser scattering } \\
\left(1 \text { to } 1000 \mu \mathrm{g} \mathrm{m}^{-3}\right) \text { by } \\
\text { using advanced particle } \\
\text { size binning }\end{array}$ & $\begin{array}{l}\text { Power supply: Yes } \\
\text { Sensor lifetime: }>8 \text { years, operating continuously for } \\
24 \mathrm{~h} / \text { day } \\
\text { Sampling mechanism: Air pump } \\
\text { Sampling interval: } 1 \mathrm{~s} \\
\text { Environmental operating conditions: } 10 \text { to } 40^{\circ} \mathrm{C} \text {; } \\
20 \% \text { to } 80 \% \\
\text { Internal data storage/wireless communication: N/S } \\
\text { Calibration: Pre-calibrated by the manufacturer }\end{array}$ \\
\hline 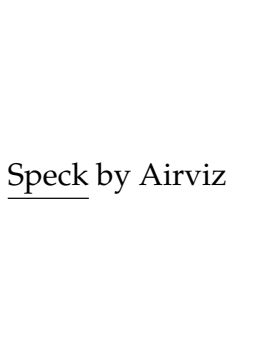 & $\begin{array}{l}\text { Detects fine PM } \\
\text { (between } 0.5 \text { and } 3.0 \\
\text { micron) in indoor } \\
\text { environments. }\end{array}$ & $\begin{array}{l}\text { Equipped with an } \\
\text { optical sensor } \\
\text { (DSM501A) that counts } \\
\text { the number of particles } \\
\text { per litre of air (ppl). It } \\
\text { can estimate the particle } \\
\text { mass per cubic meter of } \\
\text { air } \\
\left(\mu \mathrm{g} \mathrm{m}^{-3}\right) \text {. }\end{array}$ & $\begin{array}{l}\text { Power supply: Micro USB, } 5 \mathrm{~V} 500 \mathrm{~mA} \\
\text { Sampling interval: } 5 \mathrm{~s} \text { to } 4 \text { min (default } 1 \mathrm{~min} \text { ) } \\
\text { Environmental operating range: }-10 \sim+65{ }^{\circ} \mathrm{C} ;<95 \% \\
\text { Internal data storage/wireless communication: } \\
\text { Yes/Yes } \\
\text { Calibration: Pre-calibrated by the manufacturer by } \\
\text { exposing to two controlled particle concentrations }\end{array}$ \\
\hline
\end{tabular}

Multipurpose units designed for IAQ

\begin{tabular}{|c|c|c|c|}
\hline Sensor name & Pollutants & Technology & Specific Practical Features \\
\hline$\frac{+\mathrm{IQAir}}{\text { Pro }}-$ AirVisual & $\begin{array}{l}\text { A handheld device that } \\
\text { provides real-time } \\
\text { measurements of } \mathrm{PM} \\
(0.3-2.5 \mu \mathrm{m}) \text { and } \mathrm{CO}_{2} \\
(400-10,000 \text { ppm). }\end{array}$ & $\begin{array}{l}\text { Use a light-scattering } \\
\text { method to measure PMs. }\end{array}$ & $\begin{array}{l}\text { Battery: Rechargeable Li:Ion (up to } 4 \text { h on a single } \\
\text { charge) } \\
\text { Screen Size: } 5 \text { " light-emitting diode (LED) } \\
\text { Sampling mechanism: Air pump } \\
\text { Sampling interval: N/S } \\
\text { Operating temp.: } 0 \text { to } 40{ }^{\circ} \mathrm{C} ; 0 \text { to } 95 \% \\
\text { Internal data storage/wireless communication: } \\
\text { No/Yes } \\
\text { Calibration: Pre-calibrated by the manufacturer }\end{array}$ \\
\hline$\underline{\text { Air Fruit }}$ & $\begin{array}{l}\text { A handheld device that } \\
\text { provides real-time } \\
\text { measurements of } \mathrm{PM}_{2.5} \text {, } \\
\mathrm{CO}_{2} \text {, temp. and } \mathrm{RH} \text {. }\end{array}$ & $\begin{array}{l}\text { Use light-scattering to } \\
\text { measure } \mathrm{PM}_{2.5} \text {. } \\
\mathrm{PM}_{2.5}: 0 \sim 500 \mu \mathrm{g} \mathrm{m}^{-3} \\
\mathrm{CO}_{2}: 0 \sim 10,000 \mathrm{ppm}^{-0}\end{array}$ & $\begin{array}{l}\text { Power supply: } 5 \mathrm{~V} \text { USB cable } \\
\text { Sampling mechanism: Air pump } \\
\text { Detection time interval: daytime } 15 \mathrm{~min} / \text { night } 1 \mathrm{~h} \\
\text { Sampling interval: N/S } \\
\text { Environmental operating conditions: up to } 70{ }^{\circ} \mathrm{C} \text {; } \\
<100 \% \\
\text { Internal data storage/wireless communication: } \\
\text { No/Yes } \\
\text { Calibration: Pre-calibrated by the manufacturer }\end{array}$ \\
\hline
\end{tabular}


Table 3. Cont.

\begin{tabular}{|c|c|c|c|}
\hline \multicolumn{4}{|c|}{ Single-Purpose Units Designed for IAQ } \\
\hline Sensor name & Pollutant & Technology & Specific Practical Features \\
\hline$\frac{\text { Air Quality Egg }}{\text { V2 } 2020}$ & $\begin{array}{l}\text { Used for measurements } \\
\text { of } \mathrm{CO}_{2}, \mathrm{SO}_{2}, \mathrm{CO}, \mathrm{O}_{3}, \\
\mathrm{PM}\left(\mathrm{PM}_{1}, \mathrm{PM}_{2.5} \text { and }\right. \\
\left.\mathrm{PM}_{10}\right) \text { and } \mathrm{NO}_{2} \text { (not all } \\
\text { together). Each set of } \\
\text { sensors also monitors } \\
\text { temp., pressure and } \mathrm{RH} \text {. }\end{array}$ & $\begin{array}{l}\text { Dual Plantower } \\
\text { PMS5003 sensor ranges } \\
\text { between } 0.3 \text { and } 10 \mu \mathrm{m} \text {. }\end{array}$ & $\begin{array}{l}\text { Power supply: No battery, } 5 \text { V USB or Micro-USB } \\
\text { Sampling mechanism: Air pump } \\
\text { Sampling interval: N/S } \\
\text { Environmental operating conditions: up to } 40{ }^{\circ} \mathrm{C} \text {; up } \\
\text { to } 95 \% \\
\text { Sensor response time: Maximum of } 30 \mathrm{~s} \\
\text { Sensor lifetime: } 3 \text { years } \\
\text { Internal data storage/wireless communication: } \\
\text { Yes/Yes } \\
\text { Calibration: For gases, using previously calibrated } \\
\text { electrochemical gaseous sensors }\end{array}$ \\
\hline Airthings Wave Plus & $\begin{array}{l}\text { A smart air quality } \\
\text { monitor capable of } \\
\text { measuring temp., } \mathrm{RH}, \\
\text { TVOCs, air pressure, } \\
\text { radon and } \mathrm{CO}_{2} \text {. }\end{array}$ & $\begin{array}{l}\text { Sensor specifications } \\
\text { (except for } \mathrm{CO}_{2} \text { which is } \\
\text { NDIR) are not included } \\
\text { in the product sheet. } \\
\text { Settling time: } \\
\text { TVOC } \sim 7 \text { days } \\
\mathrm{CO}_{2} \sim 7 \text { days }\end{array}$ & $\begin{array}{l}\text { Battery: Yes, } 2 \text { AA } 1.5 \mathrm{~V} \\
\text { Power supply: No } \\
\text { Sampling mechanism: N/S (diffusion for radon) } \\
\text { Sampling interval: } 5 \mathrm{~min} \\
\text { Environmental operating conditions: } 4 \text { to } 40{ }^{\circ} \mathrm{C} \text {; } \\
<85 \% \\
\text { Internal data storage/ wireless communication: } \\
\text { No/Yes (Bluetooth or AirthingsSmartLink) } \\
\text { Calibration: Pre-calibrated by the manufacturer }\end{array}$ \\
\hline
\end{tabular}

Real-time measurements of $\mathrm{PM}_{1}, \mathrm{PM}_{2.5}$ and $\mathrm{PM}_{10}$ in $\mu \mathrm{g} \mathrm{m}^{-3}$. It also provides temp., $\mathrm{RH}$, pressure, $\mathrm{CO}, \mathrm{CH}_{2} \mathrm{O}$ and TVOC measurements.

AirThinx IAQ Holds Conformitè Europëenne (CE), Federal Communications Commission (FCC), PCS Type Certification Review Board (PTCRB) certificates.
Equipped with a factory calibrated Plantower PMS5003 laser particle counter.

$\mathrm{CO}_{2}: 0 \sim 3000 \mathrm{ppm}$

PMs: 0 500 $\mu \mathrm{g} \mathrm{m}^{-3}$

$\mathrm{CH}_{2} \mathrm{O}: 0 \sim 1 \mathrm{mg} \mathrm{m}^{-3}$

TVOC: 1 30 ppm of

$\mathrm{EtOH}$
Power supply: Yes (5 V DC)

Sampling mechanism: Air pump

Sampling interval: 1, 5, 10, 15, and $30 \mathrm{~min}$

Environmental operating conditions: up to $75^{\circ} \mathrm{C}$

Internal data storage/wireless communication:

No/Yes (incl. cellular)

Calibration: Pre-calibrated by the manufacturer of temp., $\mathrm{RH}, \mathrm{CO}_{2}$,

$\underline{\text { Awair }}$ $\mathrm{PM}_{2.5}$ and chemicals (VOC). It needs Wi-Fi for setup.
Real-time measurements
Senso specs:

Temp. -40 to $125{ }^{\circ} \mathrm{C}$

RH 0 to $100 \%$

$\mathrm{CO}_{2}$ 400-5000 ppm

$\mathrm{PM}_{2.5}$ : 0-1000 $\mu \mathrm{g} \mathrm{m}^{-3}$

VOCs: 0-60,000 ppb

\section{Battery: No}

Power supply: $5 \mathrm{~V} / 2.0$ A external power adapter Sampling mechanism: N/S

Sampling interval: $5 \mathrm{~min}$

Environmental operating conditions: N/S Internal data storage/wireless communication: No/Yes

Calibration: Pre-calibrated by the manufacturer

Power supply: Yes (Non-detachable USB cable)

Sampling mechanism: Air pump

Sampling interval: $5 \mathrm{~min}$

standalone air quality monitor to measure $\mathrm{T}(0$

Blueair Aware to $\left.50{ }^{\circ} \mathrm{C}\right), \mathrm{RH}(25 \%$ to $75 \%), \mathrm{CO}_{2}$ (450 to 5000

$\mathrm{N} / \mathrm{R}$

$\mathrm{ppb}), \mathrm{PM}_{2.5}$ (1 to $500 \mu \mathrm{g}$

$\mathrm{m}^{-3}$ ), and TVOC (125 to $1000 \mathrm{ppb})$.
Environmental operating conditions: 0 to $50{ }^{\circ} \mathrm{C} ; 5$ to $95 \%$

Internal data storage/wireless communication:

No/Yes

Calibration: Pre-calibrated by the manufacturer 
Table 3. Cont.

\begin{tabular}{|c|c|c|c|}
\hline \multicolumn{4}{|c|}{ Single-Purpose Units Designed for IAQ } \\
\hline Sensor name & Pollutant & Technology & Specific Practical Features \\
\hline$\underline{\text { Edimax }}$ & $\begin{array}{l}\text { The Edimax Edigreen } \\
\text { Home sensor measures } \\
\mathrm{PM}_{2.5} \text { and } \mathrm{PM}_{10} \text { in } \mu \mathrm{g} \\
\mathrm{m}^{-3}, \mathrm{CO}_{2}, \mathrm{HCHO}, \\
\text { TVOC, temp. and } \mathrm{RH} \text {. }\end{array}$ & $\begin{array}{l}\text { Use a Plantower } \\
\text { PMS5003 laser particle } \\
\text { counter, which comes } \\
\text { factory calibrated. } \\
\mathrm{PM}_{2.5 / 10}: 0-500 \mu \mathrm{g} \mathrm{m}^{-3} \\
\mathrm{PM}_{10}: 0-500 \mu \mathrm{g} \mathrm{m}^{-3} \\
\mathrm{CO}_{2}: 400-2000 \mathrm{ppm} \\
\text { TVOC: } 0-1000 \mathrm{ppb} \\
\mathrm{HCHO}: 0-1 \mathrm{mg} \mathrm{m}^{-3}\end{array}$ & $\begin{array}{l}\text { Power supply: Yes (USB power adapter) } \\
\text { Sampling mechanism: Air pump } \\
\text { Sampling interval: N/S } \\
\text { Environmental operating conditions: } 0 \text { to } 50{ }^{\circ} \mathrm{C} ; 0 \text { to } \\
100 \% \\
\text { Internal data storage/wireless communication: } \\
\text { No/Yes } \\
\text { Calibration: Pre-calibrated by the manufacturer } \\
\text { (CO } \mathrm{CO}_{2} \text { and TVOC sensors require up to } 72 \mathrm{~h} \text { to } \\
\text { self-calibrate after the installation). }\end{array}$ \\
\hline Huma-i (HI-300A) & $\begin{array}{l}\text { Advanced Portable Air } \\
\text { Quality Monitor Indoor } \\
\text { and Outdoor Measures } \\
\text { temp., } \mathrm{RH}, \mathrm{CO}_{2}, \mathrm{VOC} \text {, } \\
\mathrm{PM}_{1}, \mathrm{PM}_{2.5} \text {, and } \mathrm{PM}_{10} \text {. }\end{array}$ & $\begin{array}{l}\mathrm{CO}_{2}(400 \sim 5000 \mathrm{ppm}) \\
\mathrm{VOC}(0.000 \sim 10 \mathrm{ppm}) \\
\mathrm{PM}_{1}, \mathrm{PM}_{2.5} \text { and } \mathrm{PM}_{10} \\
\left(0 \sim 1000 \mu \mathrm{g} \mathrm{m}^{-3}\right) \\
\text { CE and FCC certification }\end{array}$ & $\begin{array}{l}\text { Battery: Yes, built-in Li-polymer @ } 650 \mathrm{mAh} / 3.7 \mathrm{~V} \\
\text { Power supply: AC 100/240 V, 50/60 HZ, USB-C } \\
\text { Sampling mechanism: Air pump } \\
\text { Sampling interval: N/S } \\
\text { Environmental operating conditions: }-10 \sim 60{ }^{\circ} \mathrm{C} \text {; } \\
0 \sim 99 \% \\
\text { Internal data storage/wireless communication: Yes } \\
\text { (90 days)/Yes } \\
\text { Calibration: Pre-calibrated by the manufacturer }\end{array}$ \\
\hline$\underline{\text { IDEAL AS10 }}$ & $\begin{array}{l}\text { The IDEAL AS10 indoor } \\
\text { air sensor measures the } \\
\text { air composition, indoor } \\
\text { climate and possible } \\
\text { environmental impacts, } \\
\text { all in real time. }\end{array}$ & $\begin{array}{l}\text { It measures } \mathrm{PM}_{2.5} \text { and } \\
\mathrm{PM}_{10}\left(0-1000 \mu \mathrm{g} \mathrm{m}^{-3}\right) \text {, } \\
\text { VOCs }(0-32,768 \mathrm{ppb}) \text {, } \\
\text { temp }\left(-10 \text { to }+50{ }^{\circ} \mathrm{C}\right) \text {, } \\
\mathrm{RH}(20-90 \%) \text { and air } \\
\text { pressure }(20-110 \mathrm{hPa}) \text {. }\end{array}$ & $\begin{array}{l}\text { Battery: No } \\
\text { Power supply: } 5 \mathrm{~V} \text { micro USB cable } \\
\text { Sampling mechanism: N/S } \\
\text { Sampling interval: } 1 \mathrm{~s} \\
\text { Transmission interval: } 60 \mathrm{~s} \\
\text { Environmental operating conditions: Up to } 50{ }^{\circ} \mathrm{C} \text {; } \\
20 \sim 90 \% \\
\text { Internal data storage/wireless communication: } \\
\text { No/Yes } \\
\text { Calibration: Pre-calibrated by the manufacturer } \\
\text { valid for } 24 \text { months }\end{array}$ \\
\hline $\begin{array}{l}\text { Laser Egg } \\
+ \text { chemical or } \\
+\mathrm{CO}_{2}\end{array}$ & $\begin{array}{l}\text { A handheld device that } \\
\text { provides real-time } \\
\text { measurements of temp., } \\
\mathrm{RH}, \mathrm{PM}_{2.5} \text { and VOCs or } \\
\mathrm{CO}_{2} \text {. }\end{array}$ & $\begin{array}{l}\text { Use light-scattering to } \\
\text { measure particles } \\
\text { between } 0.3 \text { and } 2.5 \\
\text { micron. }\end{array}$ & $\begin{array}{l}\text { Battery: Yes } \\
\text { Battery Life: } 8 \mathrm{~h} \\
\text { Power supply: DC } 5 \mathrm{~V} \text { (USB charging cable) } \\
\text { Sampling mechanism: Air pump } \\
\text { Sampling interval: N/S } \\
\text { Environmental operating conditions: N/S } \\
\text { Internal data storage/wireless communication: } \\
\text { No/Yes } \\
\text { Calibration: Pre-calibrated by the manufacturer }\end{array}$ \\
\hline $\begin{array}{l}\text { Magnasci SRL - } \\
\text { uRADMonitor A3 } \\
\text { (HW105) }\end{array}$ & $\begin{array}{l}\text { Measures } 8 \text { air quality } \\
\text { parameters including } \\
\mathrm{PM}_{2.5}, \mathrm{CO}_{2}, \mathrm{VOC}, \\
\mathrm{HCHO} \text {, temp., } \mathrm{RH}, \\
\text { barometric pressure and } \\
\text { Gamma/X-ray radiation. }\end{array}$ & $\begin{array}{l}\text { Use laser scattering } \\
\text { sensor to detect PMs; a } \\
\text { NDIR sensor to measure } \\
\mathrm{CO}_{2} \text {; an EC for HCHO, a } \\
\text { Bosch BME } 680 \text { sensor } \\
\text { for temp., RH, } \\
\text { barometric pressure and } \\
\text { VOC; and an S129BG } \\
\text { Geiger Tube to detect } \\
\text { gamma and X-ray } \\
\text { radiation. }\end{array}$ & $\begin{array}{l}\text { Battery: No } \\
\text { Power supply: } 6-28 \mathrm{~V} \\
\text { Sampling mechanism: Air pump for active flow } \\
\text { Sampling interval: N/S } \\
\text { Environmental operating conditions: up to } 85^{\circ} \mathrm{C} \text {, up } \\
\text { to } 100 \% \\
\text { Internal data storage/wireless communication: } \\
\text { No/Yes } \\
\text { Calibration: Pre-calibrated by the manufacturer }\end{array}$ \\
\hline
\end{tabular}


Table 3. Cont.

\begin{tabular}{|c|c|c|c|}
\hline \multicolumn{4}{|c|}{ Single-Purpose Units Designed for IAQ } \\
\hline Sensor name & Pollutant & Technology & Specific Practical Features \\
\hline $\begin{array}{l}\text { SainSmart - Pure } \\
\text { Morning P3 }\end{array}$ & $\begin{array}{l}\text { Measures } \mathrm{PM}_{2.5}, \mathrm{HCHO} \text {, } \\
\left.\mathrm{CO}_{2} \text {, air temp. (in }{ }^{\circ} \mathrm{C}\right) \\
\text { and } \mathrm{RH}(\%) \text {. }\end{array}$ & $\begin{array}{l}\text { Equipped with a } \\
\text { Plantower PMS5003 } \\
\text { laser particle counter. }\end{array}$ & $\begin{array}{l}\text { Battery: No } \\
\text { Power supply: Yes }(5 \mathrm{~V}) \\
\text { Sampling mechanism: Air pump } \\
\text { Sampling interval: N/S } \\
\text { Environmental operating conditions: N/S } \\
\text { Internal data storage/wireless communication: } \\
\text { No/Yes } \\
\text { Calibration: Pre-calibrated by the manufacturer }\end{array}$ \\
\hline Temtop M2000 & $\begin{array}{l}\text { Measures real-time } \\
\text { reading of } \mathrm{HCHO}, \\
\mathrm{PM}_{2.5}, \mathrm{PM}_{10} \\
\mathrm{CO}_{2} \text {, temp. and } \mathrm{RH} \text {. }\end{array}$ & $\begin{array}{l}\mathrm{PM}_{2.5} \text { range: } \\
0 \sim 999 \mu \mathrm{g} \mathrm{m}^{-3} \\
\mathrm{PM}_{10} \text { range: } \\
0 \sim 999 \mu \mathrm{g} \mathrm{m}^{-3} \\
\mathrm{CO}_{2} \text { range: } \\
0 \sim 5000 \text { ppm } \\
\text { HCHO range: } \\
0 \sim 5 \mathrm{mg} \mathrm{m}^{-3}\end{array}$ & $\begin{array}{l}\text { Battery: Yes } \\
\text { Power supply: } 5 \text { V DC } \\
\text { Sampling mechanism: Air pump } \\
\text { Sampling interval: N/S } \\
\text { Environmental operating conditions: up to } 50{ }^{\circ} \mathrm{C} \text {; } \\
<90 \% \\
\text { Internal data storage/wireless communication: } \\
\text { Yes/No } \\
\text { Calibration: Pre-calibrated by the manufacturer }\end{array}$ \\
\hline$\underline{\mathrm{uHoO}}$ & $\begin{array}{l}\text { Carries eight dedicated se } \\
\mathrm{ppb}), \mathrm{PM}_{2.5}(0-200 \mu \mathrm{g} \mathrm{m} \\
(400-10,000 \mathrm{ppm}), \mathrm{O}_{3}(10- \\
\left.85{ }^{\circ} \mathrm{C}\right), \mathrm{RH}(0 \text { to } 100 \%) \text { an } \\
\text { mbar }) .\end{array}$ & $\begin{array}{l}\text { sors for VOCs }(10-10,000 \\
), \mathrm{CO}(0-1000 \mathrm{ppm}), \mathrm{CO}_{2} \\
10,000 \mathrm{ppb}) \text {, temp. }(-40 \text { to } \\
\text { air pressure }(300-1100\end{array}$ & $\begin{array}{l}\text { Battery: No } \\
\text { Power supply: Yes, } 5 \text { V DC } \\
\text { Sampling mechanism: Air pump } \\
\text { Sampling interval: N/S } \\
\text { Internal data storage/wireless communication: } \\
\text { No/Yes } \\
\text { Calibration: Pre-calibrated by the manufacturer }\end{array}$ \\
\hline
\end{tabular}

* Designated by US EPA as a Federal Equivalent Method (FEM: EQOA-0815-227). Note 1: Sensors' lifetime of the reviewed kits is not reported in the technical documents. Note 2: N/S and N/R stand for not specified and not reported, respectively.

\section{Deployment Strategies}

Enclosed environments such as homes trap more polluted air than open environments due to the presence of indoor sources, lack of free-flow air circulation and inadequate ventilation. In addition, different exposure levels to indoor air pollutants have been reported for individuals even at the same location [32,74]. Unfortunately, the use of conventional monitoring devices are unable to satisfactorily capture a spatial variation and map instantaneous changes in IAQ because of the associated cost, non-scalability, and lack of spatio-temporal mapping of indoor air pollutants [11,30,75,76]. Considering these potentials and demands for technologies, the emergence of LCSs has changed the landscape of IAQ monitoring systems, where specific sensors and sensor-based products are manufactured and designed for indoor applications (see Tables 2 and 3, respectively). Although air pollution sensors have some drawbacks (Table 2), relatively smaller changes in the environmental parameters and less complexity of indoor air flow patterns compared to outdoor environments could be beneficial for using them indoors. Table 4 summarises examples of the sensor applications in indoor environments, in which less attention has been given to strategies for spatio-temporal distribution of multiple air pollutants. The objectives of the reviewed studies were limited to the performance evaluation of sensors in enclosed environments, in which near-source air pollution monitoring systems or considering an adult's breathing height as a common practice among the studies is inadequate to assess overall IAQ [35,77-79]. Considering indoor arrangements and the relationship between indoor-outdoor environments [80], here we focus our efforts in developing suitable strategies for indoor environments, while air pollution sensors are playing the major role in covering the area. 
Table 4. Some recent LCS deployments indoors across the world.

\begin{tabular}{|c|c|c|c|c|c|c|c|c|}
\hline Pollutants/Range of Operation & Sensor Type & Room Size & Reference Instrument & $\begin{array}{l}\text { Sensor Placement/ } \\
\text { Country }\end{array}$ & $\begin{array}{l}\text { Sampling } \\
\text { Frequency }\end{array}$ & $\begin{array}{c}\text { Correlation Factor } \\
\mathbf{R}^{2} / \text { Agreement }\end{array}$ & Duration & References \\
\hline $\mathrm{PM}_{1}, \mathrm{PM}_{2.5}, \mathrm{PM}_{10}$ & $\begin{array}{l}\text { PM-Model-II particle } \\
\text { counters (Plantower } \\
\text { PMS3003) }\end{array}$ & $88 \mathrm{~m}^{2}$ & $\begin{array}{l}\text { Particle monitor (Thermo } \\
\text { Scientific Model FH } 62 \text { C14) }\end{array}$ & $\begin{array}{l}\text { An apartment in } \\
\text { Beijing }\end{array}$ & $\begin{array}{l}1 \mathrm{~min} \\
\text { resolu- } \\
\text { tion }\end{array}$ & $\begin{array}{l}79 \% \text { of the } \\
\text { spatiotemporal } \\
\text { variation based on a } \\
\text { regression model }\end{array}$ & 10 days & [81] \\
\hline $\mathrm{PM}_{1}, \mathrm{PM}_{2.5}, \mathrm{PM}_{10}$ & $\begin{array}{l}\text { Dylos DC1100 Pro and } \\
\text { Plantower PMS sensor } \\
\text { (AirU) }\end{array}$ & $\begin{array}{l}\text { Two homes, } \\
306.6 \mathrm{~m}^{2} \text { and } \\
140 \mathrm{~m}^{2}\end{array}$ & $\begin{array}{l}\text { GRIMM, DustTrak, and } \\
\text { MiniVol }\end{array}$ & $\begin{array}{l}\text { Two households in } \\
\text { Salt Lake City, USA }\end{array}$ & $1 \mathrm{~min}$ & $\begin{array}{l}\text { See various } R^{2} \text { in the } \\
\text { manuscript }\end{array}$ & $\begin{array}{l}\text { One-week } \\
\text { calibration, several } \\
\text { weeks for sampling }\end{array}$ & [82] \\
\hline $\begin{array}{l}\mathrm{PM}_{2.5} \text { and } \mathrm{PM}_{10} \text { from } 21 \text { common } \\
\text { residential sources }\end{array}$ & $\begin{array}{l}\text { Air Quality Egg 2018; } \\
\text { IQAir AirVisual Pro (AVP); } \\
\text { Awair 2nd Edition; } \\
\text { Kaiterra Laser Egg; } \\
\text { PurpleAir Indoor; and Ikair }\end{array}$ & $\mathrm{N} / \mathrm{R}$ & $\begin{array}{l}\text { Grimm Mini Wide-Range } \\
\text { Aerosol Spectrometer Model } \\
1371\end{array}$ & $\mathrm{~N} / \mathrm{R}$ & $5 \mathrm{~min}$ & $\mathrm{R}^{2} \geq 0.83$ & $48 \mathrm{~h}$ & [83] \\
\hline $\begin{array}{l}\mathrm{CO}(0-29), \mathrm{CO}_{2}(0-3600), \mathrm{PM}_{10 / 2.5} \\
(0-1) \text { and } \operatorname{VOC}(0-46)^{*}\end{array}$ & $\begin{array}{l}\text { Aeroqual Series } 500 \text { with } \\
\text { different sensor heads. } \\
\text { gas-sensitive } \\
\text { electrochemical (GSE), } \\
\text { NDIR, laser particle } \\
\text { counter, and PID types }\end{array}$ & $\begin{array}{l}\text { Floor area of } \\
\text { merely } 9.3 \mathrm{~m}^{2}\end{array}$ & Not applicable & $\begin{array}{l}\text { Subdivided unit } \\
\text { (SDU) in Hong Kong }\end{array}$ & $\begin{array}{l}60,120 \\
5, \text { and } \\
30 \mathrm{sec}\end{array}$ & $\begin{array}{l}\text { No significant } \\
\text { correlation between } \\
\text { indoor and outdoor } \\
\text { pollutants in case of } \\
\mathrm{CO}(3.58 \%), \mathrm{PM}_{10} \\
(0.96 \%) \text {, and } \mathrm{PM}_{2.5} \\
(7.11 \%) .\end{array}$ & $\begin{array}{l}48 \mathrm{~h} \text { in each SDU in } \\
\text { the summer of } 2018\end{array}$ & [84] \\
\hline $\begin{array}{l}\text { Noise }(35-120 \mathrm{~dB}), \mathrm{T}\left(0-50^{\circ} \mathrm{C}\right), \mathrm{RH} \\
(0-100 \%), \mathrm{CO}(0-1000 \mathrm{ppm}), \mathrm{CO}_{2} \\
(0-5000 \mathrm{ppm}), \mathrm{NO}(0-20 \mathrm{ppm}), \\
\mathrm{NO}_{2}(0-20 \mathrm{ppm}) \text { and } \mathrm{PM}_{2.5} \\
(0.38-17 \mu \mathrm{m})\end{array}$ & $\begin{array}{l}\text { Netatmo Weather Station, } \\
\text { Onset Temperature, } \\
\text { Alphasense (COB4, NOB4, } \\
\text { NO2B43F, and OPC-N2), } \\
\text { and Harvard miniPEM }\end{array}$ & $\begin{array}{l}\text { In a residential } \\
\text { building }\end{array}$ & $\begin{array}{l}\text { RTI MicroPEM for PM only ( } 5 \\
\text { min avg.) }\end{array}$ & $\begin{array}{l}\text { Boston, MA, } \\
\text { USA }\end{array}$ & $1 \mathrm{~min}$ & $\begin{array}{l}\text { Carried out only for } \\
\text { PM }_{2.5} \\
\text { (Lab (TSI SidePak }{ }^{\mathrm{TM}} \\
\text { AM510): } \mathrm{R}^{2}=0.47 ; \\
\text { field (RTI MicroPEM): } \\
\mathrm{R}^{2}=0.83 \text { ) }\end{array}$ & $\begin{array}{l}\text { Multiple 1-week } \\
\text { sessions }\end{array}$ & [71] \\
\hline $\begin{array}{l}\mathrm{CO}, \mathrm{NO}_{2}, \mathrm{NO}, \mathrm{O}_{3}, \mathrm{PM}_{2.5}(\mathrm{PAM}, \\
\text { Model AS520) }\end{array}$ & $\begin{array}{l}\text { 4-electrode ECs } \\
\text { (Great Notley, UK): CO-A4 } \\
\text { (for } \mathrm{CO}), \mathrm{NO}_{2} \text {-A43F (for } \\
\mathrm{NO}_{2} \text { ), NO-A4 (for } \mathrm{NO} \text { ), } \\
\text { and Ox-A431 (for } \mathrm{O}_{3} \text { ). } \\
\text { For PM: a miniaturised } \\
\text { OPC (OPC-N }{ }_{2} \text {, } \\
\text { Alphasense) }\end{array}$ & $\begin{array}{l}\text { A living } \\
\text { room }\end{array}$ & $\begin{array}{l}\text { BLUME instrumentation uses } \\
\text { chemiluminescence to measure } \\
\mathrm{NO}_{2} \text { and NO, UV absorption } \\
\text { for } \mathrm{O}_{3} \text {, non-dispersive infrared } \\
\text { absorption for CO, and } \\
\text { particle light-scattering for } \\
\mathrm{PM}_{2.5} \text { (model EDM180, Grimm } \\
\text { Aerosol Technik, Ainring, } \\
\text { Germany). }\end{array}$ & $\begin{array}{l}\text { The indoor } \\
\text { instrument was } \\
\text { placed into the } \\
\text { home's living room, } \\
\text { which was either } \\
\text { adjacent to the back } \\
\text { garden or separated } \\
\text { from it by a room in } \\
\text { between. }\end{array}$ & $\begin{array}{l}\text { Maximum: } \\
1 \mathrm{~Hz} . \\
\text { Minimum: } \\
20 \mathrm{sec} \\
(\mathrm{PM})\end{array}$ & $\begin{array}{l}\text { For the inorganic } \\
\text { gases }\left(0.92<R^{2}<\right. \\
0.96) \\
\text { for } \mathrm{PM}_{2.5}\left(\mathrm{R}^{2}=0.64\right)\end{array}$ & $\begin{array}{l}\text { Simultaneous } \\
\text { indoor pollutant } \\
\text { measurements } \\
\text { in residential } \\
\text { buildings in Berlin, } \\
\text { Germany. } \\
\text { Instruments } \\
\text { measured one } \\
\text { week per location. }\end{array}$ & [85] \\
\hline $\begin{array}{l}\text { Ammonia (1-500 ppm), } \mathrm{CH}_{4} \\
(>1000 \mathrm{ppm}), \mathrm{C}_{3} \mathrm{H}_{8}(>1000 \mathrm{ppm}), \\
\mathrm{C}_{4} \mathrm{H}_{10}(>1000 \mathrm{ppm}), \mathrm{CO}(1-1000 \\
\text { ppm), Ethanol (10-500 ppm), } \mathrm{H}_{2} \\
\text { (1-1000 ppm), } \mathrm{NO}_{2}(0.05-10 \mathrm{ppm})\end{array}$ & MOx sensors (MICS series) & $\begin{array}{l}\text { Real-time IAQ } \\
\text { monitoring in } \\
\text { a home using } \\
\text { iAir }\end{array}$ & $N / R$ & $\begin{array}{l}\text { Guarda, } \\
\text { Portugal }\end{array}$ & $30 \mathrm{sec}$ & $\mathrm{N} / \mathrm{R}$ & $\mathrm{N} / \mathrm{R}$ & [86] \\
\hline
\end{tabular}


Table 4. Cont.

\begin{tabular}{|c|c|c|c|c|c|c|c|c|}
\hline $\begin{array}{c}\text { Pollutants/Range of } \\
\text { Operation }\end{array}$ & Sensor Type & Room Size & Reference Instrument & $\begin{array}{l}\text { Sensor Placement/ } \\
\text { Country }\end{array}$ & $\begin{array}{l}\text { Sampling } \\
\text { Frequency }\end{array}$ & $\begin{array}{c}\text { Correlation Factor } \\
\mathbf{R}^{2} / \text { Agreement }\end{array}$ & Duration & References \\
\hline $\mathrm{CO}$ and $\mathrm{PM}_{2.5}$ & $\begin{array}{l}\text { HAPEX and TZOA-R for } \mathrm{PM}_{2.5} \\
\text { EL-USB-C for CO }\end{array}$ & $\begin{array}{l}1 \mathrm{~m} \text { from an } \\
\text { indoor } \\
\text { fireplace and } \\
0.6 \mathrm{~m} \text { above } \\
\text { the ground }\end{array}$ & $\begin{array}{l}\text { DustTrak DRX (Model 8534) } \\
\text { BGI/Mesa Labs pump (Model } \\
\text { BGI4004) } \\
\text { Q-Trak (Model 7575) }\end{array}$ & $\begin{array}{l}\text { Non-smoking } \\
\text { private single-family } \\
\text { house/Spain }\end{array}$ & $5 \mathrm{~min}$ & $\mathrm{R}^{2}$ up to 85 & 5 days & [87] \\
\hline $\mathrm{CO}$ and $\mathrm{PM}_{2.5}$ & $\begin{array}{l}\text { HAPEX }\left(\mathrm{PM}_{2.5}\right) \text { and } \\
\text { EL-USB-C }(\mathrm{CO})\end{array}$ & $\begin{array}{l}\text { Main living } \\
\text { area at least at } \\
1 \mathrm{~m} \text { above the } \\
\text { ground }\end{array}$ & $\begin{array}{l}\text { SKC pump (Model Universal } \\
\text { PCXR8) }\end{array}$ & $\begin{array}{l}4 \text { households located } \\
\text { in } 4 \text { villages/India } \\
\text { March-April } 2016\end{array}$ & $5 \mathrm{~min}$ & $N / R$ & 1 week & [87] \\
\hline $\begin{array}{l}\mathrm{PM}_{2.5}\left(25 \mathrm{\mu g} \mathrm{m}^{-3}\right), \mathrm{tVOC} \\
(300 \mathrm{ppb}), \mathrm{CO}_{2}(1300 \mathrm{ppm}), \\
\mathrm{T}\left(40^{\circ} \mathrm{C}\right) \text {, and } \mathrm{RH}(60 \%)\end{array}$ & Foobot kit & $\begin{array}{l}\text { An occupied } \\
\text { bedroom (floor } \\
\text { area } \\
10.5 \mathrm{~m}^{2} \text { ) of a } \\
\text { modern flat }\end{array}$ & $\begin{array}{l}\text { GrayWolf TG-502 TVOC, } \\
\text { IQ-410, and PC-3016A }\end{array}$ & Glasgow, UK & $5 \mathrm{~min}$ & $\begin{array}{l}\text { A significant } \\
\text { agreement with the } \\
\text { GrayWolf } \\
\mathrm{T}\left(\mathrm{r}_{\mathrm{s}}=0.83-0.87\right) \\
\mathrm{RH}\left(\mathrm{r}_{\mathrm{s}}=0.94-0.95\right) \\
\mathrm{tVOC}\left(\mathrm{r}_{\mathrm{s}}=0.83-0.87\right) \\
\mathrm{PM}_{2.5}\left(\mathrm{r}_{\mathrm{s}}=0.79-0.87\right)\end{array}$ & $\begin{array}{l}81 \text { h } 25 \text { min (from } \\
28 \text { August } 23: 50 \mathrm{LT} \\
\text { to } 1 \text { September } \\
201711: 25 \mathrm{LT})\end{array}$ & [88] \\
\hline $\begin{array}{l}\mathrm{PM}, \mathrm{CO}, \mathrm{O}_{3}, \mathrm{NO}_{2} \text {, noise, } \\
\text { temp. } \\
\text { and } \mathrm{RH}\end{array}$ & $\begin{array}{l}\text { A dust sensor (Sharp, Model } \\
\text { DN7C3CA006, Osaka, Japan) } \\
\text { A 4-electrode CO sensor } \\
\text { (Alphasense, Model CO-B4 with } \\
\text { sensor board 000-01SB-02, Essex, } \\
\text { UK) } \\
\text { A 4-electrode oxidizing gas sensor } \\
\text { (Alphasense, model OX-B431 with } \\
\text { sensor board 000-01SB-02, Essex, } \\
\text { UK) } \\
\text { A temp. and RH sensor (Adafruit, } \\
\text { model AM2302, NY, USA) } \\
\text { A custom-built noise level sensor }\end{array}$ & & $\begin{array}{l}\text { DataRAM } 1500 \text { Aerosol } \\
\text { Monitor (Thermo Fisher } \\
\text { Scientific., pDR, Shoreview, } \\
\text { MN, USA) for PM } \\
\text { Q-Trak Plus } 8552 \text { (TSI Inc., } \\
\text { Shoreview, MN, USA) for CO, } \\
\text { POM (2B Technologies Inc., } \\
\text { PO3M, Boulder, CO, USA) for } \\
\mathrm{O}_{3} \\
\text { A sound level meter (NTi } \\
\text { Audio, SLM, Schaan, } \\
\text { Liechtenstein) for noise, }\end{array}$ & $\begin{array}{l}\text { Within the } \\
\text { fabrication area of a } \\
\text { manufacturing } \\
\text { facility }\end{array}$ & $5 \mathrm{~min}$ & $\begin{array}{l}0.98 \text { to } 0.99 \text { for } \\
\text { particle mass } \\
\text { densities } \\
\text { up to } 300 \mu \mathrm{g} \mathrm{m}^{-3} \\
0.99 \text { for } \mathrm{CO} \text { up to } 15 \\
\mathrm{ppm} \text {. } \\
0.98 \text { for the oxidizing } \\
\text { gas sensor }\left(\mathrm{NO}_{2}\right) \text { over } \\
\text { the sensitive range } \\
\text { from } 20 \text { to } 180 \mathrm{ppb} \text {. } \\
1 \% \text { between } 65 \text { and } 95 \\
\text { dBA. }\end{array}$ & Three months & [89] \\
\hline PM & $\begin{array}{l}\text { Wireless PM sensor, Sharp } \\
\text { GP2Y1010AU0F }\end{array}$ & $\begin{array}{l}\text { Approximately } \\
29 \mathrm{~m}^{2} \text { of floor } \\
\text { area }\end{array}$ & $\begin{array}{l}\text { TSI Sidepak AM } 150 \text { (TSI Inc., } \\
\text { Minnesota, USA) }\end{array}$ & $\begin{array}{l}2 \text { kitchens in Raipur, } \\
\text { India }\end{array}$ & $\begin{array}{l}\text { Sidepak } \\
1 \mathrm{~Hz} \\
\text { and } \\
\text { sensors } \\
0.25 \mathrm{~Hz}\end{array}$ & 0.71 & $\begin{array}{l}\text { Multiple days at } \\
\text { the two } \\
\text { households }\end{array}$ & [35] \\
\hline $\begin{array}{l}\text { Light }(0.1 \text { to } 40,000 \text { Lux }), \mathrm{T} \\
\left(-55-80^{\circ} \mathrm{C}\right), \mathrm{RH}(0-100 \%) \\
\mathrm{CO}_{2}(0-10,000 \mathrm{ppm})\end{array}$ & $\begin{array}{l}\text { TAOS TSL2561, Onset HOBO (NTC } \\
\text { thermistor, Sensirion SHT15), } \\
\text { SenseAir K-30 }\end{array}$ & $\begin{array}{l}\text { IAQ in an } \\
\text { educational } \\
\text { building }\end{array}$ & $\mathrm{N} / \mathrm{R}$ & $\begin{array}{l}\text { Two locations at } \\
\text { Illinois Institute of } \\
\text { Technology in } \\
\text { Chicago, IL }\end{array}$ & $1 \mathrm{~min}$ & $N / R$ & 1 week & [33] \\
\hline
\end{tabular}

Automatically control building climate control systems (air purifiers, kitchen hoods, bathroom or whole houg
higher than acceptable levels. ${ }^{*}$ Concentration values are in $\mathrm{mg} \mathrm{m}^{-3}$. Note: $\mathrm{N} / \mathrm{R}$ represents not reported. 
Air quality sensors should be deployed systematically across a location in order to (i) optimise the cost and the number of sensors according to building layout, space and room features, (ii) ensure the reliability level of the sensor network in case of sensor failure, (iii) provide acceptable spatial and temporal coverage of indoor air pollutants, and (iv) minimise the cost associated to computational analysis and prediction models [23,90-92] There are common suggestions regarding deployment strategies in practical engineering applications, such as sensor selection as per common indoor sources, considering the impacts of outdoor air pollution on indoors, and deploying sensors along the wall with proper accessibility for calibration or maintenance $[35,93]$. However, sensor deployment strategies, especially for IAQ applications are usually determined based on objective functions and sensor applications [90,94]. In general, deployment strategies in indoor environments vary with time and space, which can be categorised into (i) engineering, and (ii) optimisation methods. In the engineering method, previous experiences and rules of thumb are incorporated. Uniform deployment of several sensors in space would be a common practice in engineering methods as can be seen in studies listed in Table 4, which may result in a fairly expensive and unfeasible output in some cases. Application of this method may result in lack of (i) spatio-temporal mapping, (ii) controlling the response time, and (iii) generalisability to multiple rooms/spaces $[90,94,95]$. To compensate for the limitations of this method, the optimisation method has recently developed, in which indoor airflow patterns in the deployment of sensors are taken into account [96-100]. In this method, modelling tools such as computational fluid dynamics (CFD), zonal model, and multi-zone airflow model are utilised along with genetic algorithm, artificial neural networks (ANNs), simulated annealing, and stochastic approximation methods to optimise objective, cost, or fitness functions based on the predefined goals [97,98,101,102]. Although this method could bring precision in choosing the optimal strategy, optimisation methods could be computationally intensive in the large deployment of sensors in multi-zone airflow and CFD-based simulations [95]. Nevertheless, to achieve optimal strategies regardless of sensor locations and to avoid occasional error in the prediction results of small sensor networks (a combination of 3 to 4 sensors as reported by Ren and Cao [79]), systematic sensor deployment methods, such as clustering model of fuzzy C-means (FCM) algorithm based on ANN [103] or based on the genetic algorithm [104] for the efficient prediction of indoor environments could be employed.

Although no standard values for IAQ exist and the idea of setting guideline values $[13,105]$ is not new, we propose a simple deployment strategy for LCS deployment in typical indoor spaces after building the evidence-base from the relevant published literature (Figure 2). This basic strategy could be considered as a generalised plan, where developing an optimisation model is not computationally feasible and could include (i) deployment of environmental and pollutant sensors across the indoor space, whereas deploying height has to be set according to occupants' height; and (ii) deploying sensors based on specifications discussed in Figure 2, in locations, where taking samples using sensors' induction fan can represent the entire environment. In the absence of a legislative framework for regulating IAQ, such a strategy could help optimise the sampling that is representative of indoor environments and can be beneficial in planning appropriate mitigation steps for reducing the exposure from indoor air pollutants. However, an optimised network of air pollution LCSs needs to be supported by the appropriate data processing (Section 6) and predictive modelling (Section 7) to allow its interpretation, visualisation and conveying the meaningful messages to the users in a simple form. 


\begin{tabular}{|c|c|c|c|}
\hline \multicolumn{3}{|c|}{$\begin{array}{l}\text { Bedrooms (adult/kids): PMs and aerosols from dust, dust mites, bacteria } \\
\text { and viruses; } \mathrm{CO}_{2} \text { from exhaling; TVOCs from personal care products, such } \\
\text { as perfumes, hair spray, nail polish, upholstery, furniture, and carpet. }\end{array}$} & $\begin{array}{l}\text { Toilet/bathroom/laundry: TVOCs from cleaning products; } \\
\mathrm{CO}_{2} \text { from exhaling; Mould and mildew, bacteria, odorous } \\
\text { and other microbial pathogens (their formations can be } \\
\text { limited by controlling ventilation and } \mathrm{T} / \mathrm{RH} \text { ). }\end{array}$ \\
\hline $\begin{array}{l}\text { Outdoor } \\
\text { resusper }\end{array}$ & $\begin{array}{l}\mathrm{PM}_{2.5} / \mathrm{PM}_{10} \text { from } \\
\text { ion; } \mathrm{NO}_{2} / \mathrm{CO} \text { from } \\
\text { Suggested ranges }\end{array}$ & $\begin{array}{l}\text { aust emissions, and dust } \\
\text { rning/combustion; } \mathrm{O}_{3} \text { (groun }\end{array}$ & $\begin{array}{l}\text { Kitchen: } \mathrm{CO}, \mathrm{NO}_{2} \text {, PMs from cooking } \\
\text { activities; TVOCs from cleaning products; } \\
\mathrm{CO}_{2} \text { from exhaling. }\end{array}$ \\
\hline $\begin{array}{r}\text { Temp. } \\
\text { RH } \\
\text { Noise } \\
\text { Light } \\
\mathrm{PM}_{2.5} \\
\mathrm{NO}_{2} \\
\mathrm{CO}\end{array}$ & $\begin{array}{l}0 \text { to } 50^{\circ} \mathrm{C} \\
0 \text { to } 100 \% \\
0-80 \mathrm{~dB} \\
0-17,000 \text { lux } \\
0-500 \mu \mathrm{g} \mathrm{m}^{-3} \\
0-20 \mathrm{ppm}^{-} \\
0-1000 \mathrm{ppm}\end{array}$ & $\begin{array}{l}\text { and light. } \\
\text { Home office: TVOCs from } \\
\text { paint, electronic devices and } \\
\text { office equipment. } \\
\text { Home parking: } \mathrm{PM}_{2.5} / \mathrm{NO}_{2} / \mathrm{CO} \\
\text { from cars in idling mode; }\end{array}$ & $\begin{array}{l}\text { Living room (with wood-burning stove): } \mathrm{PM} \\
\text { from combustion and smoke, pet hair and } \\
\text { dander; } \mathrm{NO}_{2} / \mathrm{CO} \text { from burning/combustion; } \\
\mathrm{CO}_{2} \text { from exhaling and cooking; } \\
\text { TVOCs/formaldehyde from carpets, varnishes, } \\
\text { paints, glues, furniture, air fresheners. }\end{array}$ \\
\hline $\begin{array}{r}\mathrm{CO}_{2} \\
\mathrm{TVOCS}^{\mathrm{O}_{3}} \\
\text { Radon }\end{array}$ & $\begin{array}{l}0-5000 \mathrm{ppm}^{*} \\
0-2000 \mathrm{ppb}^{*} \\
0-20 \mathrm{ppm}^{-3} \\
\text { Up to } 50 \mathrm{~K} \mathrm{~Bq} \mathrm{~m}^{-3}\end{array}$ & $\begin{array}{l}\text { TVOCs from stored paints, } \\
\text { solvents, pesticides, } \\
\text { herbicides. }\end{array}$ & $\begin{array}{r}\text { *Optimum range for TVOCs is obtained from reviewed studies. However, } \\
\text { a higher upper limit of } 20 \mathrm{ppm} \text { with } 10 \mathrm{ppb} \text { resolution is recommended by } \\
\text { professional grade instruments for indoor applications. }\end{array}$ \\
\hline
\end{tabular}

Figure 2. Schematic diagram of a simple home deployment strategy for LCSs as per location, including proposed environmental and air pollution sensors (green boxes) and their associated ranges (a blue box) in a typical indoor space. The representative image of a home building was obtained from free sources using Google image search engine.

\section{Data Processing}

A network of LCSs requires a substantial amount of pre- and post-processing of data before presenting to the users. Pre-processed data is recorded by LCSs, which utilises an initial calibration (pre-processed data). Post-processed data is transmitted by the sensor to a database, which subsequently undergoes QA/QC protocols before being made available to the users. Pre-processed data should often not be made available to the users until sufficient QA/QC has been performed. QA/QC is essential in LCS monitoring systems and refers to a set of activities and measures that are taken to ensure that the requirements, objectives and established quality standards with a pre-established level of performance and confidence being met. However, their role is not to guarantee that the data is of the highest possible quality, which is often unreachable and unfeasible. What is sought is to ensure that the data are accurate, reliable, fit and adequate for a particular purpose or application.

\subsection{Pre-Processing of Low-Cost Sensor (LCS) Data}

LCSs are manufactured to measure numerous parameters, including but not limited to (i) date/time; (ii) environmental parameters (e.g., temperature $\left({ }^{\circ} \mathrm{C}\right)$, relative humidity ( $\mathrm{RH}, \%)$, barometric pressure); (iii) gaseous pollutants (concentration by molar ratio or mass); and (iv) particle concentrations, segregated size fractions in different size bins $\left(\mu \mathrm{g} \mathrm{m}^{-3}\right)$. The amount of data produced by LCSs is often orders of magnitude greater than traditional measurement techniques. For example, at an acquisition rate of $1 \mathrm{~Hz}$, the total number of measurements could be 86,400 per day per single measurement. If one considers monitoring of six indoor parameters at a minute sampling frequency, it will have 8640 samples in one day per location. Considering a network with multiple locations, it brings challenges to data management and processing [106-108].

Handling of large volume datasets requires an infrastructure to process data. To do so, several tools have been developed to address the processing of such large multivariable dataset, with good performance. One of the available tools is the Apache Spark framework (https://spark.apache.org; accessed on 21 March 2021), which was initially designed to be open-source. The tool supports the processing of large amounts of data using distributed computing for the development of iterative algorithms (like machine learning and graph models), interactive data mining, streaming and time-series applications [109]. The framework supports a set of programming languages such as Java, Python, Scala, and $\mathrm{R}$, while being capable of distributing data and computation with a robust fault tolerance 
mechanism for both. One of the main current tasks of this emerging smart computing platform includes the processing and streaming of large amounts of data from sensors as well as machine learning tasks $[109,110]$. This framework is able to offer an optimal model in terms of both processing time and least error rate in working with air quality databases, especially related to smart monitoring [111-114].

On top of big data frameworks-a descriptor for very large and multivariate timeseries datasets produced by LCS systems-different kinds of tool were designed to tackle specific applications. In the internet of things (IoT) area, multivariate time-series are continuously needed to be pre-processed to guarantee its fitness for their expected usage. Currently, the combination of big data frameworks along with time-series databases, data collectors, data monitors, and data visualisers, has boosted the ability to use data from LCSs to generate useful and reliable IAQ information. For time-series database management and streaming, the open-source InfluxDB platform [115] offers a variety of tools and mechanisms to deal with LCSs time-series datasets $[82,116,117]$. The capability of open-source InfluxDB has been proved for its time-series functionality, keeping costs as low as possible, making querying archives simple, and connectivity to data collectors like Telegraf and to graphing software like Grafana low-effort [116]. The Telegraf tool [118] offers a plugin architecture that supports the connection between a broad range of data sources to collect and report metrics and events. Grafana [119] has emerged as one of the most used platforms by the industry, offering a rich and extendable web interface to build dashboards on top of data sources and collectors, catch errors and monitor readings, bring compatibility with several languages, tools and frameworks [116].

In summary, pre-processed datasets always involve three problems: the quality of data, high dimensionality, and the growing amount of data. The measurements provided by LCSs are only useful when these issues are overcome. With an increase in interest surrounding big data and its applications, many open-source frameworks have been developed as discussed with the capability to process and store large amounts of timedependent data. These tools help LCS networks to effectively propagate and batch-process data enabling users to conduct a wide range of experiments concurrently with real-time monitoring of the results.

\subsection{Post-Processing of LCS Data}

Post-processing techniques, such as outlier detection, data cleaning and gap-filling methodologies could help to determine missing, duplicated, inconsistent datasets, and eliminate high-frequency noises to improve the quality of measured data [120,121]. To meet the demands for higher data quality in LCS systems, Mahajan and Kumar [106] presented a toolbox, known as Sense Your Data: Sensor toolbox. This web-based tool provides easy and efficient functions to analyse air pollution data for both researchers as well as the general public. The tool offers data plotter (including data summary), anomaly/outlier removal and gap-filling. The three different algorithms implemented in this tool for data processing are: (i) autoregressive integrated moving average (ARIMA) additive for tasks related to prediction/forecasting [122]; (ii) K-nearest neighbour (K-NN) for anomaly detection [120,123]; and (iii) the ANN model for air pollution time-series data dealing with forecasting [122] and gap-filling [120]. The two algorithms for gap filling are: (i) Interpolation using the "imputeTS" package [124] to fill the missing values in the dataset; and (ii) Kalman filter to estimate past, present and future values even when the precise nature of the system is unknown [125].

Other anomaly detection techniques that are specialised in time-series data are the SAX algorithm (symbolic aggregate approximation); [126]) and the cluster-based algorithm for anomaly detection in time-series using Mahalanobis distance (C-AMDATS; [127]). SAX addresses the detection of anomalies in time-series datasets using the concept of discords, which transforms a time-series into a sequence of characters (i.e., a string) using clustering techniques [128]. C-AMDATS, in turn, is an unsupervised learning technique that uses clustering methods and the covariance matrix to compute the Mahalanobis 
distance, to determine how a certain pattern differs from the others, and to calculate the most anomalous using an anomaly rank index. It is a multivariate technique and its performance has been evaluated as the best results compared to the SAX algorithm using urban air pollution data [127]. Recently, a lightweight python library called Luminol for time series data analysis was developed, which implements several anomaly detection algorithms [129]. Luminol owns a series of applications ranging from detecting and correcting network anomalies - the amount of writing, requests, etc.- to health, sensors and IoT applications, which could be valuable and important for post-processing timeseries data from networked LCSs.

\section{Predictive Modelling}

Developing a predictive model that can forecast the changes in IAQ and occupants' exposure is crucial to obtain concentration profiles of air pollutants in indoor spaces [130]. Predictive modelling is a commonly used technique, which employs analysis of historical/current data and generation of a model to help predicting future outcomes. With the availability of IAQ data collected using the LCSs, sophisticated techniques can be employed to develop a predictive model. In the subsequent sections, we review and consolidate the techniques used for predictive modelling and bring the prevalent best practice and knowledge to develop optimal indoor models, in which previously discussed topics are used as the foundation in the model development.

\subsection{Types of Indoor Air Quality (IAQ) Predictive Models}

IAQ modelling is a non-invasive and inexpensive method to better estimate spatiotemporal distribution of indoor air pollutants. IAQ is commonly predicted using mechanistic (white box) or statistical (black box) models. Mechanistic models utilise detailed input parameters which apply fate and transport of indoor air pollutants via diffusion, convective mass transfer, and sorption of pollutants. Mechanistic models can be applied on unoccupied microenvironments where detailed indoor/outdoor target air pollutants, building layout and ventilation conditions are available or under-controlled. Mechanistic models have been implemented in several studies to predict indoor PMs [131-133] as well as VOCs [134-137]. Mechanistic models can be categorised as single compartment mass balance-based model and CFD model, as described here:

- The single compartment mass balance-based model is a common mechanistic model that has been widely used in studies to explore IAQ with proper validation against real-world data [138-140]. Liu and Zhai [94] integrated a probability-based adjoint inverse method into the single compartment mass balance-based model to back-track indoor pollution sources. In the model, interpolation was used to obtain the pollutant concentrations at the locations among sensors, where sensor readings are assumed to be always accurate. However, this is not the right assumption in the case of LCSs due to drift error. For example, the uncompensated drift error and standard deviation of a VOC sensor in many environments were about 0.8 and 0.3 ppm per 4 months, respectively [141]. Therefore, Xiang et al. [142] improved the mass balance-based model by considering LCS specifications and optimally compensating drift errors. The corrected model was composed of an optimal indoor concentration prediction and estimation model, which was supported by a hybrid sensor network synthesis algorithm.

- CFD is a well-known mechanistic model that is restrictive in nature due to its exceptional complexity and dependency on many assumptions, approximations, and real observations. Empirical models can be integrated into detailed mathematical models to enhance the accuracy of predictions. CFD supported models by empirical/physicsbased models require additional resources and pre-existing knowledge during model development [143-145].

In statistical models, model parameters are identified using experimental data and the model structure is inferred by applying statistical methods. While mechanistic and 
empirical/physics-based models are complicated to develop and there are no established mechanisms, statistical models can help especially in case of dealing with large datasets [146]. This technique can deliver reliable outcomes, but the complete lack of physical insights is a significant drawback. Statistical models have been developed in which they appear to be less resource-demanding compared to other models. In fact, statistical models need the use of consistent input data streams via data loggers or pollution monitors, thereby, the absence of input information flow could endanger the accuracy of the model $[144,147,148]$.

In addition to traditional statistical models, such as kriging or Gaussian process regression, the use of machine-learning techniques gained increased attention in statistical IAQ predictions. The common statistical machine learning-based models are multiple linear regression, partial least squares, generalized linear model, decision trees (classification and regression trees), Bayesian hierarchical model, generalized boosting model, support vector machine, random forests, and ANN [72,146,149-153]. Although discussing the details of these methods is not the primary objective of this study, we showcase the most applicable models that can be of use in building predictive models using LCSs.

Linear regression is a statistical method that captures the linear relationships of independent variables to predict the value of a dependent variable, such as forecasting air pollution $[154,155]$. Partial least square model and generalised linear model provide a general framework for handling regression models for normal/non-normal data that can be applied in IAQ applications [156,157]. Decision trees are simple but successful techniques that predict the target value via learning simple decision rules [152,158]. Bayesian hierarchical modelling is a statistical model that utilises Bayes' theorem for estimations. The hierarchical approach facilitates the understanding of multi-parameter problems and developing computational strategies $[159,160]$. A generalised boosting model is a combination of decision tree-based algorithms and boosting techniques, which frequently fit decision trees to improve the accuracy of the model [152]. Support vector machine regression is the proposed method to deal with non-linear problems [72,161]. Random forest or random decision forests regression model is a simple, flexible and most used machine learning algorithm, which can be utilised in both classification and regression applications [162-165]. ANN is the most commonly used machine learning technique for solving complex problems [70,72,166-169]. ANN has shown the capability of estimating IAQ with an acceptable range of $0.62<\mathrm{R}^{2}<0.79$ only with one hidden layer $[167,170,171]$. However, there are few emerging applications of deep neural networks (DNN), like recurrent neural networks (RNN), long short-term memory (LSTM), and gated recurrent units (GRU), that need exploration [146,172-174].

\subsection{Best-Fit Approaches for LCS IAQ Modelling}

Table 5 presents a summary of modelling studies for residential settings that various machine learning techniques are used for the prediction of IAQ parameters. The development of machine learning and statistical models in recent years (Section 7.1) has offered significant benefits in the prediction of complex indoor environments [72,146,165]. The development of these predictive models would require large scale data collection provided by the sensor network, adequate computing infrastructure for data processing, analysis and model construction. Although the applications of predictive models are vast, the limited efforts on implementation of ANN, multiple linear regression, and random forest regression models showed acceptable performances in predicting indoor variables. Nevertheless, further efforts should be undertaken to enhance the performance of these tools in predicting all known indoor air pollutants (Figure 2) using continuously generated data by sensor networks rather than focusing only on proxies. 
Table 5. A summary of recent IAQ modelling studies that use various machine learning techniques for the prediction of IAQ parameters. Studies carried out in offices, schools and commercial buildings are excluded as their layouts could be different from residential buildings.

\begin{tabular}{|c|c|c|c|c|c|c|}
\hline $\begin{array}{l}\text { Input to the } \\
\text { Model }\end{array}$ & $\begin{array}{l}\text { Model } \\
\text { Output }\end{array}$ & $\begin{array}{c}\text { Environment, Location, } \\
\text { Date, Time }\end{array}$ & Model & Correlation & Recommendations & Reference \\
\hline $\begin{array}{l}\text { Time, in/out } \\
\text { temp. and } \\
\text { RH }\end{array}$ & $\begin{array}{l}\text { Temp. and } \\
\text { RH }\end{array}$ & $\begin{array}{l}\text { A test house }\left(147.8 \mathrm{~m}^{2} \times\right. \\
3 \mathrm{~m}) \text { in Finland }\end{array}$ & $\begin{array}{l}\text { Artificial neural } \\
\text { networks or ANN } \\
\text { (non-linear } \\
\text { autoRegressive with } \\
\text { eXternal input } \\
\text { (NNARX) model and } \\
\text { genetic algorithm were } \\
\text { employed to construct } \\
\text { networks) }\end{array}$ & $\begin{array}{l}\text { Correlation } \\
\text { coefficients } \\
0.998 \text { and } \\
0.997 \text { for } \\
\text { temp. and } \\
\text { RH }\end{array}$ & $\begin{array}{l}\text { Three-layer feed-forward } \\
\text { ANN is capable of } \\
\text { predicting any nonlinear } \\
\text { relations even in a complex } \\
\text { situation where (i) some } \\
\text { impact factors are still } \\
\text { unclear and (ii) some } \\
\text { important information is } \\
\text { unavailable. }\end{array}$ & [175] \\
\hline $\begin{array}{l}\text { Resident } \\
\text { activities }\end{array}$ & $\mathrm{CO}_{2}$ & $\begin{array}{l}\text { Two smart apartments } \\
\text { and a smart workplace, } \\
\text { Washington State } \\
\text { University, Pullman, WA, } \\
\text { USA }\end{array}$ & $\begin{array}{l}\text { Naïve Bayes, ANN and } \\
\text { Decision tree }\end{array}$ & $\mathrm{N} / \mathrm{S}$ & $\begin{array}{l}\text { The decision tree algorithm } \\
\text { did perform best in many } \\
\text { examined cases. }\end{array}$ & {$[176]$} \\
\hline $\begin{array}{l}\text { Indoor temp. } \\
\text { and RH }\end{array}$ & $\mathrm{CO}_{2}$ & $\begin{array}{l}8 \text { apartments ( } 4 \\
\text { bedrooms and } 6 \text { living } \\
\text { rooms) located in } \\
\text { Kuopio, Finland, from } \\
\text { May to October } 2011 . \\
\text { Measurements were } \\
\text { taken every } 10 \mathrm{~s} .\end{array}$ & $\begin{array}{l}\text { ANN (based on } \\
\text { multilayer perceptron } \\
\text { network) }\end{array}$ & $\begin{array}{l}\mathrm{R}^{2} \leq 0.39 \pm \\
0.02\end{array}$ & $\begin{array}{l}\text { The prediction of } \mathrm{CO}_{2} \text { is } \\
\text { difficult, if it is based only } \\
\text { on measurements of } \mathrm{RH} \\
\text { and temp. }\end{array}$ & [168] \\
\hline $\begin{array}{l}\text { Temp., } \\
\text { internal } \\
\mathrm{PM}_{2.5} \\
\text { sources, } \\
\text { window } \\
\text { opening }\end{array}$ & $\mathrm{PM}_{2.5}$ & $\begin{array}{l}\text { Dwelling (single-storey } \\
\text { flats in England during } \\
\text { October to May) }\end{array}$ & ANN (feed-forward) & $\begin{array}{l}\mathrm{R}^{2} \text { between } \\
0.84 \text { to } 0.90\end{array}$ & $\begin{array}{l}\text { ANN is able to accurately } \\
\text { predict IAQ from a reduced } \\
\text { set of input variables. }\end{array}$ & [177] \\
\hline $\begin{array}{l}\text { Temp. and } \\
\text { RH }\end{array}$ & $\mathrm{CO}_{2}$ & $\begin{array}{l}\text { Two rooms named as } \\
\text { R203 and R204 of Smart } \\
\text { Home }\end{array}$ & $\begin{array}{l}\text { Decision tree } \\
\text { regression/random } \\
\text { forest }\end{array}$ & $\begin{array}{l}\text { Accuracy of } \\
46.25 \text { ppm }\end{array}$ & $\begin{array}{l}\text { It is possible to use the } \\
\text { Random Forest method } \\
\text { with sufficient accuracy in } \\
\mathrm{CO}_{2} \text { estimation on the } \\
\text { basis of the internal and } \\
\text { external temp./RH, the } \\
\text { time and date as the input } \\
\text { parameters. }\end{array}$ & [178] \\
\hline $\begin{array}{l}\text { Particle } \\
\text { deposition } \\
\text { parameters }\end{array}$ & $\mathrm{PM}_{2.5}$ & $\mathrm{~N} / \mathrm{R}$ & $\begin{array}{l}\text { ANN (multilayer } \\
\text { Perceptron) }\end{array}$ & $\mathrm{N} / \mathrm{R}$ & $\begin{array}{l}\text { ANN gives an average } \\
\text { relative error of }<5 \%\end{array}$ & {$[179]$} \\
\hline $\begin{array}{l}\mathrm{PM}_{2.5}, \mathrm{PM}_{10} \\
\mathrm{CO}_{2} \text {, temp., } \\
\text { and } \mathrm{RH}\end{array}$ & $\begin{array}{l}\text { Indoor } \\
\text { airborne } \\
\text { culturable } \\
\text { bacteria }\end{array}$ & $\begin{array}{l}\text { Data were measured in } \\
\text { various buildings in } \\
\text { Baoding, a city that } \\
\text { suffers from } \mathrm{PM}_{2.5} \\
\text { pollution in China. }\end{array}$ & $\begin{array}{l}\text { General } \\
\text { regression } \\
\text { neural } \\
\text { network (GRNN) }\end{array}$ & $\mathrm{N} / \mathrm{R}$ & $\begin{array}{l}\text { A machine learning-based } \\
\text { method can estimate the } \\
\text { concentration of indoor } \\
\text { airborne culturable } \\
\text { bacteria. } \\
\text { A well-trained GRNN } \\
\text { model can help to quickly } \\
\text { acquire the estimated } \\
\text { concentration. }\end{array}$ & [180] \\
\hline $\begin{array}{l}\text { Ambient } \\
\mathrm{PM}_{2.5} \text { with } 10 \\
\text { and } 80 \text { min of } \\
\text { lag time }\end{array}$ & $\mathrm{PM}_{2.5}$ & $\begin{array}{l}\text { Indoor and ambient } \\
\mathrm{PM}_{2.5} \text { in } 13 \text { households } \\
\text { in Beijing, China. }\end{array}$ & Exponential regression & $\mathrm{R}^{2} \sim 0.87$ & $\begin{array}{l}\text { The } \mathrm{PM}_{2.5} \text { concentrations } \\
\text { can be predicted based on } \\
\text { ambient measurements. } \\
\text { The overall exposure } \\
\text { would be overestimated } \\
\text { without taking indoor air } \\
\text { concentrations into } \\
\text { consideration. }\end{array}$ & {$[181]$} \\
\hline
\end{tabular}


Table 5. Cont.

\begin{tabular}{|c|c|c|c|c|c|c|}
\hline $\begin{array}{l}\text { Input to the } \\
\text { Model }\end{array}$ & $\begin{array}{l}\text { Model } \\
\text { Output }\end{array}$ & $\begin{array}{c}\text { Environment, Location, } \\
\text { Date, Time }\end{array}$ & Model & Correlation & Recommendations & Reference \\
\hline $\begin{array}{l}\text { Temp. } \\
\text { and RH }\end{array}$ & $\mathrm{CO}_{2}$ & $\begin{array}{l}\text { In a room for almost a } \\
\text { week (starting from } 11 \\
\text { February 2015, to } 18 \\
\text { February 2015) in Mons, } \\
\text { Belgium }\end{array}$ & $\begin{array}{l}\text { ANN (multilayer } \\
\text { Perceptron) }\end{array}$ & $\begin{array}{l}<17 \text { ppm } \\
\text { difference on } \\
\text { average to } \\
\text { actual } \mathrm{CO}_{2} \\
\text { value }\end{array}$ & $\begin{array}{l}\text { Open-loop and } \\
\text { five-steps-ahead prediction } \\
\text { networks had better MSE } \\
\text { performances, higher } \\
\text { sensitivity and specificity } \\
\text { values vs. open- and } \\
\text { closed-loop models. } \\
\text { The } \mathrm{CO}_{2} \text { concentration } \\
\text { data are always needed to } \\
\text { obtain acceptable } \\
\text { predictions. }\end{array}$ & {$[166]$} \\
\hline $\begin{array}{l}\text { One } \\
\text { dependent } \\
\text { variable and } \\
87 \text { potential } \\
\text { predictor } \\
\text { variables }\end{array}$ & $\mathrm{PM}_{2.5}$ & $\begin{array}{l}\text { 7-day } \mathrm{PM}_{2.5} \\
\text { measurements inside the } \\
\text { homes of pregnant } \\
\text { women from January } \\
2014 \text { to December } 2015 \text { in } \\
\text { Ulaanbaatar, Mongolia }\end{array}$ & $\begin{array}{l}\text { Multiple linear } \\
\text { regression } \\
\text { and random forest } \\
\text { regression }\end{array}$ & $\begin{array}{l}\text { Multiple } \\
\text { linear } \\
\text { regression } \\
\left(\mathrm{R}^{2}=50.2 \%\right) \\
\text { and random } \\
\text { forest } \\
\text { regression } \\
\left(\mathrm{R}^{2}=48.9 \%\right)\end{array}$ & $\begin{array}{l}\text { The improved performance } \\
\text { of blended multiple linear } \\
\text { regression/random forest } \\
\text { regression models in } \\
\text { predicting indoor air } \\
\text { pollution. }\end{array}$ & [164] \\
\hline $\begin{array}{l}\mathrm{PM}_{10}, \mathrm{PM}_{2.5}, \\
\mathrm{CO}_{2} \text {, temp., } \\
\text { and RH }\end{array}$ & $\mathrm{PM}_{1}$ & $\begin{array}{l}\text { Real-time daily IAQ } \\
\text { monitoring in } 10 \\
\text { households during } \\
\text { March } 2014 \text { to July 2014, } \\
\text { India. }\end{array}$ & $\begin{array}{l}\text { Multiple linear } \\
\text { regression }\end{array}$ & $\mathrm{R}^{2}=0.81-0.98$ & $\begin{array}{l}\text { Multiple linear regression } \\
\text { models were found to } \\
\text { perform satisfactorily as } \\
\text { indicated by } 0.92<\text { index of } \\
\text { agreement }<0.99 \text { and } 0.81 \\
<\mathrm{R}^{2}<0.98 \text {. }\end{array}$ & [182] \\
\hline $\begin{array}{l}\text { Ambient } \\
\mathrm{PM}_{2.5} \text { and } \\
\text { questionnaire- } \\
\text { elicited } \\
\text { information }\end{array}$ & $\mathrm{PM}_{2.5}$ & $\begin{array}{l}\text { Daily average of } \mathrm{PM}_{2.5} \\
\text { during } 3 \text { consecutive } \\
\text { days in summer and } \\
\text { winter for } 116 \\
\text { households in Hong } \\
\text { Kong }\end{array}$ & Linear mixed regression & $\begin{array}{l}\mathrm{R}^{2}=0.61 \text { by } \\
\text { cross- } \\
\text { validation }\end{array}$ & $\begin{array}{l}\text { The fitted linear } \\
\text { mixed-effects model is } \\
\text { moderately predictive for } \\
\text { the observed indoor } \mathrm{PM}_{2.5} \text {. }\end{array}$ & [183] \\
\hline
\end{tabular}

Based on the review of various predictive modelling techniques, it has been found that the statistical models based on machine learning (Table 5) could provide a good fit for indoor air pollution prediction in smart homes. This technique provides a powerful tool for modelling the behaviour of indoor built environments with a complex interplay of the response and predictor variables. The predictive model should also be able to optimally maintain its stability in dealing with inaccurate readings and source generation rate estimates by applying proper weighting factors (a function of sensor drift and source generation rates) to improve the overall prediction accuracy. To do so, mechanistic model techniques are utilised to provide the basis for the selection of appropriate parameters for machine learning models on theoretical physics-based principles. However, uncertainty and potential disadvantages of mechanistic models as highlighted in the previous section could endanger the feasibility of the model in multiple buildings or the case of an occupied building.

\section{Conclusions and Future Remarks}

People spend a significant amount of their time in indoor environments, where they are most probably exposed to at least one IAQ problem. IAQ remains mostly unregulated and maintaining safe IAQ during the long-term stay at homes to tackle the novel coronavirus pandemic, or similar outbreaks is more challenging [184]. Smart homes equipped with air quality LCSs and integrated processing/predicting tools can offer a healthy environment to occupants. Although technologies in this field are continuously evolving, emerging knowledge among the researchers in different fields is sparse, and smart home components are considered separately due to diversity in the research field. Here, we reviewed the standard protocols needed to be met to satisfy the indoor measurement challenges. Then we 
reviewed data assimilation and data processing tools and predictive modelling techniques to estimate indoor exposure. From the study, the following conclusions are drawn:

- Indoor pollutants are released from different sources at different concentration levels, thereby, selection of LCSs should be in the way that they can serve the task according to the target pollutants and concentrations. The accuracy and diversity of LCSs used in indoor environments is an important focus in deployment strategies of LCSs in smart homes. Proper deployment height is also suggested due to variation in exposure heights among the occupants.

- Deployment of networked LCSs to map spatio-temporal distribution of indoor air pollutants is necessary to optimise the number of deployed LCSs, obtain meaningful data, reducing the computational time/cost, and data handling without losing accuracy. There are limited studies on long-term deployments of sensor networks, especially in indoor residential environments.

- The lack of data reliability and QA/QC is counted as the most important challenge associated with LCSs. We emphasised an important role of laboratory calibration of LCS. Relying only on initial LCS calibration, which is a prevalent practice in reviewed studies, for long-term deployment should be complemented by routine performance testing to the success of networked sensors. Such performance evaluations can allow maintaining data quality, oversee manufacturing variability, sensor stability, drift and ageing over time.

- $\quad$ Several open-source tools have been developed for data processing to give network providers the tools to deploy large-scale networks with little overhead. As LCSs record large amounts of time-series data, open-source tools such as InfluxDB and Grafana are necessary to be able to capture and process recorded measurements as well as allow easy visualisations for both the network operator and the occupants. Considering home-specific internal data servers can offer additional security from the external threats.

- A wide range of data processing tools are available with many capabilities, including data cleaning, data plotting and different types of anomaly detection. These tools can increase the confidence and reliability of the data, improving the services provided by the network providers and improving the experience for the occupants.

- There is an increasing trend towards the application of machine learning-based statistical models due to the availability of a continuous flow of IAQ data using LCSs. However, there are several limitations of exclusive data-based studies due to the lack of established knowledge related to the selection of desirable parameters, appropriate performance metrics, and the application of different models for different scenarios. Therefore, the best way forward would be to further advance the knowledge of statistical models for IAQ prediction by carrying out larger-scale deployments and considering a wider range of indoor pollutants that are backed by the theoretical principles from mechanistic models for modelling the underlying micro-environmental principles and mechanisms.

Making homes smarter is becoming an integral component of the smart city concept. According to the Allied Business Intelligence (ABI) Research report on smart homes [185], almost 300 million smart homes are set to be installed around the world by 2022 . Having smart homes in terms of IAQ is not a distant dream. This review reveals the benefits of using technological advancement in estimating the effects of long-term exposure to indoor air pollutants and determining new prevention strategies and control measures on health conditions in smart homes. It contributes to future generations of smart buildings as well as designing of smart cities and embracing smart technologies for IAQ monitoring by the general public and adopted in their routine lifestyle. Some of the ongoing projects such as the MyGlobalHome [186] aim to develop such advanced property development platform by connecting developers to consumers of sustainable and connected homes and seek to bridge a gap between the smart technology developers and property developers. The efforts by the aforementioned projects along with the support of ongoing research 
activities concerning air quality sensors could result in appreciable health benefits to smart home occupants.

Author Contributions: Conceptualization, P.K.; methodology, H.O. and P.K.; writing-original draft preparation, H.O. and P.K.; writing—review and editing, H.O., P.K., J.H., M.G. and E.G.S.N.; supervision, P.K.; project administration, P.K.; funding acquisition, P.K.; resources, P.K. All authors have read and agreed to the published version of the manuscript.

Funding: This research was funded by an Innovate UK funded project 'MyGlobalHome' prototype project and the pilot demonstrator under the Technology Strategy Board File References: 104782 and 106168, respectively.

Institutional Review Board Statement: Not applicable.

Informed Consent Statement: Not applicable.

Data Availability Statement: Not applicable.

Acknowledgments: We thank the reviewers for their time to help with the review of the article.

Conflicts of Interest: The authors declare no conflict of interest.

\section{References}

1. Abraham, S.; Li, X. Design of a low-cost wireless indoor air quality sensor network system. Int. J. Wirel. Inf. Netw. 2016, 23, 57-65. [CrossRef]

2. Amoatey, P.; Omidvarborna, H.; Baawain, M.S.; Al-Mamun, A. Indoor air pollution and exposure assessment of the gulf cooperation council countries: A critical review. Environ. Int. 2018, 121, 491-506. [CrossRef] [PubMed]

3. Amoatey, P.; Omidvarborna, H.; Baawain, M.S.; Al-Mamun, A.; Bari, A.; Kindzierski, W.B. Association between human health and indoor air pollution in the Gulf Cooperation Council (GCC) countries: A review. Rev. Environ. Health 2020, 35, 157-171. [CrossRef] [PubMed]

4. Koivisto, A.J.; Kling, K.I.; Hänninen, O.; Jayjock, M.; Löndahl, J.; Wierzbicka, A.; Fonseca, A.S.; Uhrbrand, K.; Boor, B.E.; Jiménez, A.S.; et al. Source specific exposure and risk assessment for indoor aerosols. Sci. Total Environ. 2019, 668, 13-24. [CrossRef] [PubMed]

5. Brittain, O.S.; Wood, H.; Kumar, P. Prioritising indoor air quality in building design can mitigate future airborne viral outbreaks. Cities Health 2020, 1-4. [CrossRef]

6. Kumar, P.; Morawska, L. Could fighting airborne transmission be the next line of defence against COVID-19 spread? City Environ. Interact. 2019, 4, 100033. [CrossRef]

7. Kumar, P.; Imam, B. Footprints of air pollution and changing environment on the sustainability of built infrastructure. Sci. Total Environ. 2013, 444, 85-101. [CrossRef] [PubMed]

8. Branco, P.T.; Alvim-Ferraz, M.C.M.; Martins, F.G.; Sousa, S.I. Quantifying indoor air quality determinants in urban and rural nursery and primary schools. Environ. Res. 2019, 176, 108534. [CrossRef]

9. Kumar, P.; Omidvarborna, H.; Pilla, F.; Lewin, N. A primary school driven initiative to influence commuting style for dropping-off and picking-up of pupils. Sci. Total Environ. 2020, 727, 138360. [CrossRef] [PubMed]

10. Salthammer, T.; Uhde, E.; Schripp, T.; Schieweck, A.; Morawska, L.; Mazaheri, M.; Clifford, S.; He, C.; Buonanno, G.; Querol, X.; et al. Children's well-being at schools: Impact of climatic conditions and air pollution. Environ. Int. 2016, 94, 196-210. [CrossRef]

11. Kumar, P.; Skouloudis, A.N.; Bell, M.; Viana, M.; Carotta, M.C.; Biskos, G.; Morawska, L. Real-time sensors for indoor air monitoring and challenges ahead in deploying them to urban buildings. Sci. Total Environ. 2016, 560, 150-159. [CrossRef]

12. Schieweck, A.; Uhde, E.; Salthammer, T.; Salthammer, L.C.; Morawska, L.; Mazaheri, M.; Kumar, P. Smart homes and the control of indoor air quality. Renew. Sustain. Energy Rev. 2018, 94, 705-718. [CrossRef]

13. WHO. World Health Organization: Guidelines for Indoor Air Quality: Selected Pollutants; World Health Organization: Geneva, Switzerland, 2010.

14. EU. 2008. Directive 2008/50/EC of the European Parliament and of the Council on Ambient Air Quality and Cleaner Air for Europe. 21 2008.L 152/1 116.2008. Available online: https:/ / eur-lex.europa.eu/eli/dir/2008/50/oj (accessed on 21 March 2021).

15. Lahrz, T.; Bischof, W.; Sagunski, H. Gesundheitliche Bewertung von Kohlendioxid in der Innenraumluft. Bundesgesundheitsblatt Gesundh. Gesundh. 2008, 51, 1358-1369.

16. WHO, World Health Organization. Air Quality Guidelines: Global Update 2005: Particulate Matter, Ozone, Nitrogen Dioxide, and Sulfur Dioxide; World Health Organization: Geneva, Switzerland, 2006.

17. Ad hoc AG. Beurteilung von Innenraumluftkontaminationen mittels Referenz- und Richtwerten. Bundesgesundheitsblatt Gesundh. Gesundh. 2007, 50, 990-1005. [CrossRef]

18. Caron, A.; Redon, N.; Thevenet, F.; Hanoune, B.; Coddeville, P. Performances and limitations of electronic gas sensors to investigate an indoor air quality event. Build. Environ. 2016, 107, 19-28. [CrossRef] 
19. Kumar, P.; Omidvarborna, H.; Barwise, Y.; Tiwari, A. Mitigating Exposure to Traffic Pollution In and Around Schools: Guidance for Children, Schools and Local Communities; Global Centre for Clean Air Research (GCARE): Guildford, UK, 2020; p. 24. [CrossRef]

20. Madrid, N.; Boulton, R.; Knoesen, A. Remote monitoring of winery and creamery environments with a wireless sensor system. Build. Environ. 2017, 119, 128-139. [CrossRef]

21. Mahajan, S.; Kumar, P.; Pinto, J.A.; Riccetti, A.; Schaaf, K.; Camprodon, G.; Smári, V.; Passani, A.; Forino, G. A citizen science approach for enhancing public understanding of air pollution. Sustain. Cities Soc. 2020, 52, 101800. [CrossRef]

22. Stocker, M.; Baranizadeh, E.; Portin, H.; Komppula, M.; Rönkkö, M.; Hamed, A.; Virtanen, A.; Lehtinen, K.; Laaksonen, A.; Kolehmainen, M. Representing situational knowledge acquired from sensor data for atmospheric phenomena. Environ. Modell. Softw. 2014, 58, 27-47. [CrossRef]

23. Rai, A.C.; Kumar, P.; Pilla, F.; Skouloudis, A.N.; Di Sabatino, S.; Ratti, C.; Yasar, A.; Rickerby, D. End-user perspective of low-cost sensors for outdoor air pollution monitoring. Sci. Total Environ. 2017, 607, 691-705. [CrossRef]

24. Morawska, L.; Thai, P.K.; Liu, X.; Asumadu-Sakyi, A.; Ayoko, G.; Bartonova, A.; Bedini, A.; Chai, F.; Christensen, B.; Dunbabin, M.; et al. Applications of low-cost sensing technologies for air quality monitoring and exposure assessment: How far have they gone? Environ. Int. 2018, 116, 286-299. [CrossRef] [PubMed]

25. Leidinger, M.; Sauerwald, T.; Alépée, C.; Schütze, A. Miniaturized integrated gas sensor systems combining metal oxide gas sensors and pre-concentrators. Procedia Eng. 2016, 168, 293-296. [CrossRef]

26. Penza, M.; EuNetAir Consortium. COST Action TD1105: Overview of sensor-systems for air-quality monitoring. Procedia Eng. 2014, 87, 1370-1377. [CrossRef]

27. Penza, M.; Hertel, O.; Spetz, A.L.; Quass, U. New sensing technologies and methods for air pollution monitoring. Urban Clim. 2015, 14, 327. [CrossRef]

28. Schütze, A.; Baur, T.; Leidinger, M.; Reimringer, W.; Jung, R.; Conrad, T.; Sauerwald, T. Highly sensitive and selective VOC sensor systems based on semiconductor gas sensors: How to? Environment 2017, 4, 20. [CrossRef]

29. Chojer, H.; Branco, P.T.B.S.; Martins, F.G.; Alvim-Ferraz, M.C.M.; Sousa, S.I.V. Development of low-cost indoor air quality monitoring devices: Recent advancements. Sci. Total Environ. 2020, 727, 138385. [CrossRef] [PubMed]

30. Kumar, P.; Martani, C.; Morawska, L.; Norford, L.; Choudhary, R.; Bell, M.; Leach, M. Indoor air quality and energy management through real-time sensing in commercial buildings. Energy Build. 2016, 111, 145-153. [CrossRef]

31. Clark, M.L.; Peel, J.L.; Balakrishnan, K.; Breysse, P.N.; Chillrud, S.N.; Naeher, L.P.; Rodes, C.E.; Vette, A.F.; Balbus, J.M. Health and household air pollution from solid fuel use: The need for improved exposure assessment. Environ. Health Perspect. 2013, 121, 1120-1128. [CrossRef] [PubMed]

32. Johnson, M.; Lam, N.; Brant, S.; Gray, C.; Pennise, D. Modeling indoor air pollution from cookstove emissions in developing countries using a Monte Carlo single-box model. Atmos. Environ. 2011, 45, 3237-3243. [CrossRef]

33. Ali, A.S.; Zanzinger, Z.; Debose, D.; Stephens, B. Open Source Building Science Sensors (OSBSS): A low-cost Arduino-based platform for long-term indoor environmental data collection. Build. Environ. 2016, 100, 114-126. [CrossRef]

34. Mead, M.I.; Popoola, O.A.M.; Stewart, G.B.; Landshoff, P.; Calleja, M.; Hayes, M.; Baldovi, J.J.; McLeod, M.W.; Hodgson, T.F.; Dicks, J.; et al. The use of electrochemical sensors for monitoring urban air quality in low-cost, high-density networks. Atmos. Environ. 2013, 70, 186-203. [CrossRef]

35. Patel, S.; Li, J.; Pandey, A.; Pervez, S.; Chakrabarty, R.K.; Biswas, P. Spatio-temporal measurement of indoor particulate matter concentrations using a wireless network of low-cost sensors in households using solid fuels. Environ. Res. 2017, 152, 59-65. [CrossRef] [PubMed]

36. Pedersen, T.H.; Nielsen, K.U.; Petersen, S. Method for room occupancy detection based on trajectory of indoor climate sensor data. Build. Environ. 2017, 115, 147-156. [CrossRef]

37. Schütze, A. Integrated sensor systems for indoor applications: Ubiquitous monitoring for improved health, comfort and safety. Procedia Eng. 2015, 120, 492-495. [CrossRef]

38. Cross, E.S.; Williams, L.R.; Lewis, D.K.; Magoon, G.R.; Onasch, T.B.; Kaminsky, M.L.; Worsnop, D.R.; Jayne, J.T. Use of electrochemical sensors for measurement of air pollution: Correcting interference response and validating measurements. Atmos. Meas. Tech. 2017, 10, 3575-3588. [CrossRef]

39. EU. Measuring Air Pollution with Low-Cost Sensors, Thoughts on the Quality of Data Measured by Sensors. Available online: https:/ / ec.europa.eu/environment/air/pdf/Brochure\%20lower-cost\%20sensors.pdf (accessed on 21 March 2021).

40. Spinelle, L.; Gerboles, M.; Kok, G.; Persijn, S.; Sauerwald, T. Review of portable and low-cost sensors for the ambient air monitoring of benzene and other volatile organic compounds. Sensors 2017, 17, 1520. [CrossRef] [PubMed]

41. Alphasense, Alphasense Application Note. AAN 803-03, 2014, 10, 3575-3588. Available online: https://zueriluft.ch/makezurich/ AAN803.pdf (accessed on 21 March 2021).

42. Lewis, A.; Peltier, W.R.; von Schneidemesser, E. Low-Cost Sensors for the Measurement of Atmospheric Composition: Overview of Topic and Future Applications. Available online: https://www.wmo.int/pages/prog/arep/gaw/documents/Low_cost_ sensors_prefinal.pdf (accessed on 21 March 2021).

43. Kohler, H.; Röber, J.; Link, N.; Bouzid, I. New applications of tin oxide gas sensors: I. Molecular identification by cyclic variation of the working temperature and numerical analysis of the signals. Sens. Actuat. B Chem. 1999, 61, 163-169. [CrossRef]

44. Herberger, S.; Herold, M.; Ulmer, H.; Burdack-Freitag, A.; Mayer, F. Detection of human effluents by a MOS gas sensor in correlation to VOC quantification by GC/MS. Build. Environ. 2010, 45, 2430-2439. [CrossRef] 
45. Liu, X.; Cheng, S.; Liu, H.; Hu, S.; Zhang, D.; Ning, H. A survey on gas sensing technology. Sensors 2012, 12, 9635-9665. [CrossRef]

46. Fine, G.F.; Cavanagh, L.M.; Afonja, A.; Binions, R. Metal oxide semi-conductor gas sensors in environmental monitoring. Sensors 2010, 10, 5469-5502. [CrossRef]

47. Kida, T.; Nishiyama, A.; Yuasa, M.; Shimanoe, K.; Yamazoe, N. Highly sensitive $\mathrm{NO}_{2}$ sensors using lamellar-structured WO3 particles prepared by an acidification method. Sens. Actuat. B Chem. 2009, 135, 568-574. [CrossRef]

48. Piedrahita, R.; Xiang, Y.; Masson, N.; Ortega, J.; Collier, A.; Jiang, Y.; Li, K.; Dick, R.P.; Lv, Q.; Hannigan, M.; et al. The next generation of low-cost personal air quality sensors for quantitative exposure monitoring. Atmos. Meas. Tech. 2014, 7, 3325-3336. [CrossRef]

49. Williams, D.E. Semiconducting oxides as gas-sensitive resistors. Sens. Actuat. B Chem. 1999, 57, 1-16. [CrossRef]

50. Spinelle, L.; Gerboles, M.; Aleixandre, M.; Bonavitacola, F. Evaluation of metal oxides sensors for the monitoring of $\mathrm{O}_{3}$ in ambient air at ppb level. Chem. Eng. Trans. 2016, 54,319-324.

51. Wolfrum, E.J.; Meglen, R.M.; Peterson, D.; Sluiter, J. Metal oxide sensor arrays for the detection, differentiation, and quantification of volatile organic compounds at sub-parts-per-million concentration levels. Sens. Actuat. B Chem. 2006, 115, 322-329. [CrossRef]

52. Hodgkinson, J.; Tatam, R.P. Optical gas sensing: A review. Meas. Sci. Technol. 2012, 24, 012004. [CrossRef]

53. Holstius, D.M.; Pillarisetti, A.; Smith, K.R.; Seto, E. Field calibrations of a low-cost aerosol sensor at a regulatory monitoring site in California. Atmos. Meas. Tech. 2014, 7, 1121-1131. [CrossRef]

54. Tong, Z.; Xiong, X.; Patra, P. Miniaturized $\mathrm{PM}_{2.5}$ particulate sensor based on optical sensing. In Proceedings of the ASEE-NE Conference, Boston, MA, USA, 30 April-2 May 2015; p. 6604.

55. Weekly, K.; Rim, D.; Zhang, L.; Bayen, A.M.; Nazaroff, W.W.; Spanos, C.J. Low-cost coarse airborne particulate matter sensing for indoor occupancy detection. In Proceedings of the 2013 IEEE International Conference on Automation Science and Engineering (CASE), Madison, WI, USA, 17-20 August 2013; pp. 32-37.

56. Clausen, C.; Han, A.; Kristensen, M.; Bentien, A. Optical sensor technology for simultaneous measurement of particle speed and concentration of micro sized particles. In Proceedings of the SENSORS, 2013 IEEE, Baltimore, MD, USA, 3-6 November 2013; pp. $1-4$.

57. Northcross, A.L.; Edwards, R.J.; Johnson, M.A.; Wang, Z.M.; Zhu, K.; Allen, T.; Smith, K.R. A low-cost particle counter as a realtime fine-particle mass monitor. Environ. Sci. Process. Impacts 2013, 15, 433-439. [CrossRef]

58. Schmidt-Ott, A.; Ristovski, Z.D. Measurement of airborne particles. Indoor Environment: Airborne Particles and Settled Dust; Wiley: Hoboken, NJ, USA, 2003; pp. 56-81.

59. Kim, J.Y.; Chu, C.H.; Shin, S.M. ISSAQ: An integrated sensing systems for real-time indoor air quality monitoring. IEEE Sens. J. 2014, 14, 4230-4244. [CrossRef]

60. Austin, E.; Novosselov, I.; Seto, E.; Yost, M.G. Laboratory evaluation of the Shinyei PPD42NS low-cost particulate matter sensor. PLOS ONE 2015, 10, 0137789.

61. Olivares, G.; Longley, I.; Coulson, G. Development of a Low-Cost Device for Observing Indoor Particle LEVELS associated with Source Activities in the Home; International Society of Exposure Science (ISES): Seattle, WA, USA, 2012.

62. Sousan, S.; Koehler, K.; Thomas, G.; Park, J.H.; Hillman, M.; Halterman, A.; Peters, T.M. Inter-comparison of low-cost sensors for measuring the mass concentration of occupational aerosols. Aerosol Sci. Technol. 2016, 50, 462-473. [CrossRef]

63. Wang, Y.; Li, J.; Jing, H.; Zhang, Q.; Jiang, J.; Biswas, P. Laboratory evaluation and calibration of three low-cost particle sensors for particulate matter measurement. Aerosol Sci. Technol. 2015, 49, 1063-1077. [CrossRef]

64. Masson, N.; Piedrahita, R.; Hannigan, M. Quantification method for electrolytic sensors in long-term monitoring of ambient air quality. Sensors 2015, 15, 27283-27302. [CrossRef] [PubMed]

65. Pang, X.; Shaw, M.D.; Lewis, A.C.; Carpenter, L.J.; Batchellier, T. Electrochemical ozone sensors: A miniaturised alternative for ozone measurements in laboratory experiments and air-quality monitoring. Sens. Actuat. B Chem. 2017, 240, 829-837. [CrossRef]

66. Williams, D.E.; Henshaw, G.S.; Bart, M.; Laing, G.; Wagner, J.; Naisbitt, S.; Salmond, J.A. Validation of low-cost ozone measurement instruments suitable for use in an air-quality monitoring network. Meas. Sci. Technol. 2013, 24, 065803. [CrossRef]

67. AQ-SPEC, Sensor List. Available online: http:/ / www.aqmd.gov/aq-spec/sensors/ (accessed on 21 March 2021).

68. Papapostolou, V.; Zhang, H.; Feenstra, B.J.; Polidori, A. Development of an environmental chamber for evaluating the performance of low-cost air quality sensors under controlled conditions. Atmos. Environ. 2017, 171, 82-90. [CrossRef]

69. US EPA. Air Sensor Toolbox; Evaluation of Emerging Air Pollution Sensor Performance. US-EPA n.d. Available online: https:/ / www.epa.gov / air-sensor-toolbox/evaluation-emerging-air-sensor-performance (accessed on 21 March 2021).

70. Spinelle, L.; Gerboles, M.; Villani, M.G.; Aleixandre, M.; Bonavitacola, F. Field calibration of a cluster of low-cost available sensors for air quality monitoring. Part A: Ozone and nitrogen dioxide. Sens. Actuat. B Chem. 2015, 215, 249-257. [CrossRef]

71. Gillooly, S.E.; Zhou, Y.; Vallarino, J.; Chu, M.T.; Michanowicz, D.R.; Levy, J.I.; Adamkiewicz, G. Development of an in-home, real-time air pollutant sensor platform and implications for community use. Environ. Pollut. 2019, 244, 440-450. [CrossRef]

72. Mahajan, S.; Kumar, P. Evaluation of low-cost sensors for quantitative personal exposure monitoring. Sustain. Cities Soc. 2020, 57, 102076. [CrossRef]

73. Omidvarborna, H.; Kumar, P.; Tiwari, A. 'Envilution ${ }^{\mathrm{TM}}{ }^{\prime}$ chamber for performance evaluation of low-cost sensors. Atmos. Environ. 2020, 223, 117264. [CrossRef]

74. Armendáriz-Arnez, C.; Edwards, R.D.; Johnson, M.; Rosas, I.A.; Espinosa, F.; Masera, O.R. Indoor particle size distributions in homes with open fires and improved Patsari cook stoves. Atmos. Environ. 2010, 44, 2881-2886. [CrossRef] 
75. Hart, J.K.; Martinez, K. Environmental sensor networks: A revolution in the earth system science? Earth Sci. Rev. 2006, 78, 177-191. [CrossRef]

76. Muste, M.V.; Bennett, D.A.; Secchi, S.; Schnoor, J.L.; Kusiak, A.; Arnold, N.J.; Mishra, S.K.; Ding, D.; Rapolu, U. End-to-end cyberinfrastructure for decision-making support in watershed management. J. Water Res. Plan. Manag. 2013, 139, 565-573. [CrossRef]

77. Kar, A.; Rehman, I.H.; Burney, J.; Puppala, S.P.; Suresh, R.; Singh, L.; Singh, V.K.; Ahmed, T.; Ramanathan, N.; Ramanathan, V. Real-time assessment of black carbon pollution in Indian households due to traditional and improved biomass cookstoves. Environ. Sci. Technol. 2012, 46, 2993-3000. [CrossRef] [PubMed]

78. Leavey, A.; Londeree, J.; Priyadarshini, P.; Puppala, J.; Schechtman, K.B.; Yadama, G.; Biswas, P. Real-time particulate and CO concentrations from cookstoves in rural households in Udaipur, India. Environ. Sci. Technol. 2015, 49, 7423-7431. [CrossRef] [PubMed]

79. Ren, J.; Cao, S.J. Incorporating online monitoring data into fast prediction models towards the development of artificial intelligent ventilation systems. Sustain. Cities Soc. 2019, 47, 101498. [CrossRef]

80. Quang, T.N.; He, C.; Morawska, L.; Knibbs, L.D. Influence of ventilation and filtration on indoor particle concentrations in urban office buildings. Atmos. Environ. 2013, 79, 41-52. [CrossRef]

81. Shen, H.; Hou, W.; Zhu, Y.; Zheng, S.; Ainiwaer, S.; Shen, G.; Chen, Y.; Cheng, H.; Hu, J.; Wan, Y.; et al. Temporal and spatial variation of $\mathrm{PM}_{2.5}$ in indoor air monitored by low-cost sensors. Sci. Total Environ. 2021, 770, 145304. [CrossRef] [PubMed]

82. Hegde, S.; Min, K.T.; Moore, J.; Lundrigan, P.; Patwari, N.; Collingwood, S.; Balch, A.; Kelly, K.E. Indoor Household Particulate Matter Measurements Using a Network of Low-cost Sensors. Aerosol Air Qual. Res. 2020, 20, 381-394. [CrossRef]

83. Wang, Z.; Delp, W.W.; Singer, B.C. Performance of low-cost indoor air quality monitors for $\mathrm{PM}_{2.5}$ and $\mathrm{PM}_{10}$ from residential sources. Build. Environ. 2020, 171, 106654. [CrossRef]

84. Cheung, P.K.; Jim, C.Y. Indoor air quality in substandard housing in Hong Kong. Sustain. Cities Soc. 2019, 48, 101583. [CrossRef]

85. Krause, A.; Zhao, J.; Birmili, W. Low-cost sensors and indoor air quality: A test study in three residential homes in Berlin, Germany. Gefahrstoffe Reinhaltung Der Luft 2019, 79, 87-92. [CrossRef]

86. Marques, G.; Pitarma, R. A cost-effective air quality supervision solution for enhanced living environments through the internet of things. Electronics 2019, 8, 170. [CrossRef]

87. Curto, A.; Donaire-Gonzalez, D.; Barrera-Gómez, J.; Marshall, J.D.; Nieuwenhuijsen, M.J.; Wellenius, G.A.; Tonne, C. Performance of low-cost monitors to assess household air pollution. Environ. Res. 2018, 163, 53-63. [CrossRef]

88. Moreno-Rangel, A.; Sharpe, T.; Musau, F.; McGill, G. Field evaluation of a low-cost indoor air quality monitor to quantify exposure to pollutants in residential environments. J. Sens. Sens. Syst. 2018, 7, 373-388. [CrossRef]

89. Thomas, G.; Sousan, S.; Tatum, M.; Liu, X.; Zuidema, C.; Fitzpatrick, M.; Koehler, K.; Peters, T. Low-cost, distributed environmental monitors for factory worker health. Sensors 2018, 18, 1411. [CrossRef] [PubMed]

90. Rackes, A.; Ben-David, T.; Waring, M.S. Sensor networks for routine indoor air quality monitoring in buildings: Impacts of placement, accuracy, and number of sensors. Sci. Technol. Built Environ. 2018, 24, 188-197. [CrossRef]

91. Tayyar, S.; Rym, B.B.; Parmantier, Y.; Fousseret, Y.; Ramdani, N. Towards optimal sensor deployment for location tracking in smart home. In Journées d'Etude sur la TéléSanté; Sorbonne Universités: Paris, France, 2019; ffhal-02161057.

92. Yang, C.T.; Chen, S.T.; Den, W.; Wang, Y.T.; Kristiani, E. Implementation of an intelligent indoor environmental monitoring and management system in cloud. Future Gener. Comput. Syst. 2019, 96, 731-749. [CrossRef]

93. Parkinson, T.; Parkinson, A.; de Dear, R. Continuous IEQ monitoring system: Context and development. Build. Environ. 2019, 149, 15-25. [CrossRef]

94. Liu, X.; Zhai, Z.J. Prompt tracking of indoor airborne contaminant source location with probability-based inverse multi-zone modeling. Build. Environ. 2009, 44, 1135-1143. [CrossRef]

95. Fontanini, A.D.; Vaidya, U.; Ganapathysubramanian, B. A methodology for optimal placement of sensors in enclosed environments: A dynamical systems approach. Build. Environ. 2016, 100, 145-161. [CrossRef] [PubMed]

96. Chen, Y.L.; Wen, J. Sensor system design for building indoor air protection. Build. Environ. 2008, 43, 1278-1285. [CrossRef]

97. Chen, Y.L.; Wen, J. Comparison of sensor systems designed using multizone, zonal, and CFD data for protection of indoor environments. Build. Environ. 2010, 45, 1061-1071. [CrossRef]

98. Chen, Y.L.; Wen, J. The selection of the most appropriate airflow model for designing indoor air sensor systems. Build. Environ. 2012, 50, 34-43. [CrossRef]

99. Liu, X.; Zhai, Z.J. Protecting a whole building from critical indoor contamination with optimal sensor network design and source identification methods. Build. Environ. 2009, 44, 2276-2283. [CrossRef]

100. Zhang, T.; Chen, Q. Identification of contaminant sources in enclosed spaces by a single sensor. Indoor Air 2007, 17, 439-449. [CrossRef] [PubMed]

101. Zeng, L.; Gao, J.; Lv, L.; Zhang, R.; Tong, L.; Zhang, X.; Huang, Z.; Zhang, Z. Markov-chain-based probabilistic approach to optimize sensor network against deliberately released pollutants in buildings with ventilation systems. Build. Environ. 2020, 168, 106534. [CrossRef]

102. Zhang, T.; Li, X.; Zhao, Q.; Rao, Y. Control of a novel synthetical index for the local indoor air quality by the artificial neural network and genetic algorithm. Sustain. Cities Soc. 2019, 51, 101714. [CrossRef] 
103. Cao, S.J.; Ding, J.; Ren, C. Sensor Deployment Strategy using Cluster Analysis of Fuzzy C-means Algorithm: Towards Online Control of Indoor Environment's Safety and Health. Sustain. Cities Soc. 2020, 59, 102190. [CrossRef]

104. Ding, Y.; Fu, X. Kernel-based fuzzy c-means clustering algorithm based on genetic algorithm. Neurocomputing 2016, 188, 233-238. [CrossRef]

105. Harrison, P.T.C. Indoor air quality guidelines. In Air Quality for People; Rooley, R.H., Sherratt, A., Eds.; Mid-Career College Press: Cambridge, UK, 2002; pp. 61-70.

106. Mahajan, S.; Kumar, P. Sense Your Data: Sensor Toolbox Manual, Version 1.0; Global Centre for Clean Air Research (GCARE): Guildford, UK, 2019. [CrossRef]

107. Abu-Elkheir, M.; Hayajneh, M.; Ali, N.A. Data management for the internet of things: Design primitives and solution. Sensors 2013, 13, 15582-15612. [CrossRef]

108. Samourkasidis, A.; Papoutsoglou, E.; Athanasiadis, I.N. A template framework for environmental timeseries data acquisition. Environ. Modell. Softw. 2019, 117, 237-249. [CrossRef]

109. Apache Spark. Apache Spark: Lightning-Fast Unified Analytics Engine. Available online: https://spark.apache.org (accessed on 21 March 2021).

110. Zaharia, M.; Xin, R.S.; Wendell, P.; Das, T.; Armbrust, M.; Dave, A.; Meng, X.; Rosen, J.; Venkataraman, S.; Franklin, M.J.; et al. Apache spark: A unified engine for big data processing. Commun. ACM 2016, 59, 56-65. [CrossRef]

111. Ameer, S.; Shah, M.A.; Khan, A.; Song, H.; Maple, C.; Islam, S.U.; Asghar, M.N. Comparative analysis of machine learning techniques for predicting air quality in smart cities. IEEE Access 2019, 7, 128325-128338. [CrossRef]

112. Asgari, M.; Farnaghi, M.; Ghaemi, Z. Predictive mapping of urban air pollution using Apache Spark on a Hadoop cluster. In Proceedings of the 2017 International Conference on Cloud and Big Data Computing, London, UK, 17-19 September 2017; pp. 89-93.

113. Rahi, P.; Sood, S.P.; Bajaj, R. Smart platforms of air quality monitoring: A logical literature exploration. In Proceedings of the International Conference on Futuristic Trends in Networks and Computing Technologies, Chandigarh, India, 22-23 November 2019; pp. 52-63.

114. Zhu, D.; Cai, C.; Yang, T.; Zhou, X. A machine learning approach for air quality prediction: Model regularization and optimization. Big Data Cogn. Comput. 2018, 2, 5. [CrossRef]

115. Influxdata. Real-Time Visibility into Stacks, Sensors and Systems. Available online: https:/ / www.influxdata.com (accessed on 21 March 2021).

116. Coleman, J.R.; Meggers, F. Sensing of Indoor Air Quality-Characterization of Spatial and Temporal Pollutant Evolution Through Distributed Sensing. Front. Built Environ. 2018, 4, 28. [CrossRef]

117. Min, K.T.; Lundrigan, P.; Sward, K.; Collingwood, S.C.; Patwari, N. Smart home air filtering system: A randomized controlled trial for performance evaluation. Smart Health 2018, 9, 62-75. [CrossRef]

118. Telegraf. Telegraf is the Open Source Server Agent to Help You Collect Metrics from Your Stacks, Sensors and Systems. Available online: https: / / www.influxdata.com/time-series-platform/telegraf (accessed on 21 March 2021).

119. Grafana. The Open Observability Platform. Available online: https://grafana.com (accessed on 21 March 2021).

120. Ottosen, T.B.; Kumar, P. Outlier detection and gap filling methodologies for low-cost air quality measurements. Environ. Sci. Process. Impacts 2019, 21, 701-713. [CrossRef]

121. Ottosen, T.B.; Kumar, P. The influence of the vegetation cycle on the mitigation of air pollution by a deciduous roadside hedge. Sustain. Cities Soc. 2020, 53, 101919. [CrossRef]

122. Mahajan, S.; Chen, L.J.; Tsai, T.C. Short-term $\mathrm{PM}_{2.5}$ forecasting using exponential smoothing method: A comparative analysis. Sensors 2018, 18, 3223. [CrossRef] [PubMed]

123. Ramaswamy, S.; Rastogi, R.; Shim, K. Efficient algorithms for mining outliers from large data sets. In Proceedings of the ACM Sigmod Record, Dallas, TX, USA, 16-18 May 2000; Volume 29, pp. 427-438.

124. Moritz, S.; Bartz-Beielstein, T. imputeTS: Time series missing value imputation in R. $R$ J. 2017, 9, 207-218. [CrossRef]

125. Welch, G.; Bishop, G. An Introduction to the Kalman Filter; Technical Report; Department of University of North Carolina: Chapel Hill, NC, USA, 2006.

126. Keogh, E.; Lin, J.; Fu, A. HOT SAX: Efficiently finding the most unusual time series subsequence. In Proceedings of the 5th IEEE International Conference on Data Mining (ICDM), Houston, TX, USA, 27-30 November 2005; pp. 226-233.

127. Nascimento, E.G.S.; Tavares, O.L.; De Souza, A.F. A cluster-based algorithm for anomaly detection in time series using mahalanobis distance. In Proceedings of the 2015 International Conference on Artificial Intelligence, ICAI 2015-WORLDCOMP 2015, Las Vegas, NV, USA, 27-30 July 2015; pp. 622-628.

128. Mohammad, Y.; Nishida, T. Robust learning from demonstrations using multidimensional SAX. In Proceedings of the 2014 14th International Conference on Control, Automation and Systems-ICCAS, Gyeonggi-do, Korea, 22-25 October 2014.

129. Luminol. Anomaly Detection and Correlation Library. Available online: https://github.com/linkedin/luminol (accessed on 21 March 2021).

130. Rajasegarar, S.; Zhang, P.; Zhou, Y.; Karunasekera, S.; Leckie, C.; Palaniswami, M. High resolution spatio-temporal monitoring of air pollutants using wireless sensor networks. In Proceedings of the 2014 IEEE Ninth International Conference on Intelligent Sensors, Sensor Networks and Information Processing (ISSNIP), Singapore, 21-24 April 2014; pp. 1-6. 
131. Chen, F.; Simon, C.M.; Lai, A.C. Modeling particle distribution and deposition in indoor environments with a new drift-flux model. Atmos. Environ. 2006, 40, 357-367. [CrossRef]

132. Hussein, T.; Kulmala, M. Indoor aerosol modeling: Basic principles and practical applications. Water Air Soil Pollut. 2008, 8, 23-34. [CrossRef]

133. Schneider, T.; Jensen, K.A.; Clausen, P.A.; Afshari, A.; Gunnarsen, L.; Wåhlin, P.; Glasius, M.; Palmgren, F.; Nielsen, O.J.; Fogh C.L. Prediction of indoor concentration of $0.5-4 \mu \mathrm{m}$ particles of outdoor origin in an uninhabited apartment. Atmos. Environ. 2004, 38, 6349-6359. [CrossRef]

134. Carslaw, N. A new detailed chemical model for indoor air pollution. Atmos. Environ. 2007, 41, 1164-1179. [CrossRef]

135. Little, J.C.; Hodgson, A.T.; Gadgil, A.J. Modeling emissions of volatile organic compounds from new carpets. Atmos. Environ. 1994, 28, 227-234. [CrossRef]

136. Liu, Z.; Ye, W.; Little, J.C. Predicting emissions of volatile and semivolatile organic compounds from building materials: A review. Build. Environ. 2013, 64, 7-25. [CrossRef]

137. Mendez, M.; Blond, N.; Blondeau, P.; Schoemaecker, C.; Hauglustaine, D.A. Assessment of the impact of oxidation processes on indoor air pollution using the new time-resolved INCA-Indoor model. Atmos. Environ. 2015, 122, 521-530. [CrossRef]

138. Chaloulakou, A.; Mavroidis, I. Comparison of indoor and outdoor concentrations of CO at a public school. Evaluation of an indoor air quality model. Atmos. Environ. 2002, 36, 1769-1781. [CrossRef]

139. Georgopoulos, P.G.; Isukapalli, S.S.; Krishnan, K. Modeling exposures to chemicals from multiple sources and routes. In Quantitative Modeling in Toxicology; John Wiley and Sons: Hoboken, NJ, USA, 2010; pp. 315-351.

140. Miller-Leiden, S.; Lohascio, C.; Nazaroff, W.W.; Macher, J.M. Effectiveness of in-room air filtration and dilution ventilation for tuberculosis infection control. J. Air Waste Manag. 1996, 46, 869-882. [CrossRef] [PubMed]

141. Ekberg, L.E. Volatile organic compounds in office buildings. Atmos. Environ. 1994, 28, 3571-3575. [CrossRef]

142. Xiang, Y.; Piedrahita, R.; Dick, R.P.; Hannigan, M.; Lv, Q.; Shang, L. A hybrid sensor system for indoor air quality monitoring. In Proceedings of the 2013 IEEE International Conference on Distributed Computing in Sensor Systems, Cambridge, MA, USA, 20-23 May 2013; pp. 96-104.

143. Clark, M.L.; Reynolds, S.J.; Burch, J.B.; Conway, S.; Bachand, A.M.; Peel, J.L. Indoor air pollution, cookstove quality, and housing characteristics in two Honduran communities. Environ. Res. 2010, 110, 12-18. [CrossRef] [PubMed]

144. Milner, J.; Vardoulakis, S.; Chalabi, Z.; Wilkinson, P. Modelling inhalation exposure to combustion-related air pollutants in residential buildings: Application to health impact assessment. Environ. Int. 2011, 37, 268-279. [CrossRef] [PubMed]

145. Srebric, J.; Vukovic, V.; He, G.; Yang, X. CFD boundary conditions for contaminant dispersion, heat transfer and airflow simulations around human occupants in indoor environments. Build. Environ. 2008, 43, 294-303. [CrossRef]

146. Wei, W.; Ramalho, O.; Malingre, L.; Sivanantham, S.; Little, J.C.; Mandin, C. Machine learning and statistical models for predicting indoor air quality. Indoor Air 2019, 29, 704-726. [CrossRef] [PubMed]

147. Chowdhury, Z.; Campanella, L.; Gray, C.; Al Masud, A.; Marter-Kenyon, J.; Pennise, D.; Charron, D.; Zuzhang, X. Measurement and modeling of indoor air pollution in rural households with multiple stove interventions in Yunnan, China. Atmos. Environ. 2013, 67, 161-169. [CrossRef]

148. Gurley, E.S.; Salje, H.; Homaira, N.; Ram, P.K.; Haque, R.; Petri Jr, W.A.; Bresee, J.; Moss, W.J.; Luby, S.P.; Breysse, P.; et al. Seasonal concentrations and determinants of indoor particulate matter in a low-income community in Dhaka, Bangladesh. Environ. Res. 2013, 121, 11-16. [CrossRef] [PubMed]

149. Bellinger, C.; Jabbar, M.S.M.; Zaïane, O.; Osornio-Vargas, A. A systematic review of data mining and machine learning for air pollution epidemiology. BMC Public Health 2017, 17, 907. [CrossRef]

150. Li, J.; Heap, A.D.; Potter, A.; Daniell, J.J. Application of machine learning methods to spatial interpolation of environmental variables. Environ. Modell. Softw. 2011, 26, 1647-1659. [CrossRef]

151. Postolache, O.A.; Pereira, J.D.; Girao, P.S. Smart sensors network for air quality monitoring applications. IEEE Trans. Instrum. Meas. 2009, 58, 3253-3262. [CrossRef]

152. Rokach, L.; Maimon, O.Z. Data Mining with Decision Trees: Theory and Applications; World Scientific Publishing Co., Pte. Ltd.: Singapore, 2015; Volume 69.

153. Symonds, P.; Taylor, J.; Chalabi, Z.; Mavrogianni, A.; Davies, M.; Hamilton, I.; Vardoulakis, S.; Heaviside, C.; Macintyre, H. Development of an England-wide indoor overheating and air pollution model using artificial neural networks. J. Build. Perform. Simul. 2016, 9, 606-619. [CrossRef]

154. Grömping, U. Variable importance assessment in regression: Linear regression versus random forest. Am. Stat. 2009, 63, 308-319. [CrossRef]

155. Luo, C.H.; Yang, H.; Huang, L.P.; Mahajan, S.; Chen, L.J. A Fast PM 2.5 Forecast approach based on time-series data analysis, regression and regularization. In Proceedings of the 2018 Conference on Technologies and Applications of Artificial Intelligence (TAAI), Taichung, Taiwan, 30 November-2 December 2018; pp. 78-81.

156. Esposito Vinzi, V.; Chin, W.W.; Henseler, J.; Wang, H. Handbook of Partial Least Squares: Concepts, Methods and Applications; Springer: Heidelberg, Germany; Dordrecht, The Netherlands; London, UK; New York, NY, USA, 2010.

157. Lee, S.; Kim, M.J.; Kim, J.T.; Yoo, C.K. In search for modeling predictive control of indoor air quality and ventilation energy demand in subway station. Energy Build. 2015, 98, 56-65. [CrossRef] 
158. Choi, M.L.; Lim, M.J.; Kwon, Y.M.; Chung, D.K. A study on the prediction method of emergency room (ER) pollution level based on deep learning using scattering sensor. J. Eng. Appl. Sci. 2017, 12, 2560-2564.

159. Mohri, M.; Rostamizadeh, A.; Talwalkar, A. Foundation of Machine Learning; Dietterich, T., Ed.; MIT Press: Cambridge, MA, USA, 2012.

160. Sarra, A.; Fontanella, L.; Valentini, P.; Palermi, S. Quantile regression and Bayesian cluster detection to identify radon prone areas J. Environ. Radioact. 2016, 164, 354-364. [CrossRef] [PubMed]

161. Balabin, R.M.; Lomakina, E.I. Support vector machine regression (SVR/LS-SVM)—an alternative to neural networks (ANN) for analytical chemistry? Comparison of nonlinear methods on near infrared (NIR) spectroscopy data. Analyst 2011, 136, 1703-1712. [CrossRef] [PubMed]

162. Kropat, G.; Bochud, F.; Jaboyedoff, M.; Laedermann, J.P.; Murith, C.; Palacios, M.; Baechler, S. Improved predictive mapping of indoor radon concentrations using ensemble regression trees based on automatic clustering of geological units. J. Environ. Radioact. 2015, 147, 51-62. [CrossRef] [PubMed]

163. Loy-Benitez, J.; Vilela, P.; Li, Q.; Yoo, C. Sequential prediction of quantitative health risk assessment for the fine particulate matter in an underground facility using deep recurrent neural networks. Ecotoxicol. Environ. Saf. 2019, 169, 316-324. [CrossRef] [PubMed]

164. Yuchi, W.; Gombojav, E.; Boldbaatar, B.; Galsuren, J.; Enkhmaa, S.; Beejin, B.; Naidan, G.; Ochir, C.; Legtseg, B.; Byambaa, T.; et al. Evaluation of random forest regression and multiple linear regression for predicting indoor fine particulate matter concentrations in a highly polluted city. Environm. Pollut. 2019, 245, 746-753. [CrossRef]

165. Zimmerman, N.; Presto, A.A.; Kumar, S.P.; Gu, J.; Hauryliuk, A.; Robinson, E.S.; Robinson, A.L.; Subramanian, R. A machine learning calibration model using random forests to improve sensor performance for lower-cost air quality monitoring. Atmos. Meas. Tech. 2018, 11, 291-313. [CrossRef]

166. Khazaei, B.; Shiehbeigi, A.; Kani, A.H.M.A. Modeling indoor air carbon dioxide concentration using artificial neural network. Int. J. Environ. Sci. Technol. 2019, 16, 729-736. [CrossRef]

167. Park, S.; Kim, M.; Kim, M.; Namgung, H.G.; Kim, K.T.; Cho, K.H.; Kwon, S.B. Predicting PM 10 concentration in Seoul metropolitan subway stations using artificial neural network (ANN). J. Hazard. Mater. 2018, 341, 75-82. [CrossRef]

168. Skön, J.P.; Johansson, M.; Raatikainen, M.; Leiviskä, K.; Kolehmainen, M. Modelling indoor air carbon dioxide $\left(\mathrm{CO}_{2}\right)$ concentration using neural network. Methods 2012, 14, 16.

169. Sofuoglu, S.C. Application of artificial neural networks to predict prevalence of building-related symptoms in office buildings. Build. Environ. 2008, 43, 1121-1126. [CrossRef]

170. Elbayoumi, M.; Ramli, N.A.; Yusof, N.F.F.M. Development and comparison of regression models and feedforward backpropagation neural network models to predict seasonal indoor $\mathrm{PM}_{2.5-10}$ and $\mathrm{PM}_{2.5}$ concentrations in naturally ventilated schools. Atmos. Pollut. Res. 2015, 6, 1013-1023. [CrossRef]

171. Kim, M.; Kim, Y.; Sung, S.; Yoo, C. Data-driven prediction model of indoor air quality by the preprocessed recurrent neural networks. In Proceedings of the 2009 ICCAS-SICE IEEE, Fukuoka, Japan, 18-21 August 2009; pp. 1688-1692.

172. Ahn, J.; Shin, D.; Kim, K.; Yang, J. Indoor air quality analysis using deep learning with sensor data. Sensors 2017, $17,2476$. [CrossRef] [PubMed]

173. Lin, Y.; Mago, N.; Gao, Y.; Li, Y.; Chiang, Y.Y.; Shahabi, C.; Ambite, J.L. Exploiting spatiotemporal patterns for accurate air quality forecasting using deep learning. In Proceedings of the 26th ACM SIGSPATIAL International Conference on Advances in Geographic Information Systems, Seattle, WA, USA, 6-9 November 2018; pp. 359-368.

174. Ma, J.; Cheng, J.C.; Lin, C.; Tan, Y.; Zhang, J. Improving air quality prediction accuracy at larger temporal resolutions using deep learning and transfer learning techniques. Atmos. Environ. 2019, 214, 116885. [CrossRef]

175. Lu, T.; Viljanen, M. Prediction of indoor temperature and relative humidity using neural network models: Model comparison. Neural. Comput. Appl. 2009, 18, 345. [CrossRef]

176. Deleawe, S.; Kusznir, J.; Lamb, B.; Cook, D.J. Predicting air quality in smart environments. J. Ambient Intell. Smart Environ. 2010, 2, 145-154. [CrossRef] [PubMed]

177. Das, P.; Shrubsole, C.; Jones, B.; Hamilton, I.; Chalabi, Z.; Davies, M.; Mavrogianni, A.; Taylor, J. Using probabilistic samplingbased sensitivity analyses for indoor air quality modelling. Build. Environ. 2014, 78, 171-182. [CrossRef]

178. Vanus, J.; Martinek, R.; Bilik, P.; Zidek, J.; Dohnalek, P.; Gajdos, P. New method for accurate prediction of $\mathrm{CO}_{2}$ in the Smart Home. In Proceedings of the 2016 IEEE International Instrumentation and Measurement Technology Conference Proceedings, Taipei, Taiwan, 23-26 May 2016; pp. 1-5.

179. Gheziel, A.; Hanini, S.; Mohamedi, B.; Ararem, A. Particle dispersion modeling in ventilated room using artificial neural network. Nucl. Sci. Tech. 2017, 28, 5. [CrossRef]

180. Li, J.; Biswas, P. Optical characterization studies of a low-cost particle sensor. Aerosol Air Qual. Res. 2017, 17, 1691-1704. [CrossRef]

181. Qi, M.; Zhu, X.; Du, W.; Chen, Y.; Chen, Y.; Huang, T.; Pan, X.; Zhong, Q.; Sun, X.; Zeng, E.Y.; et al. Exposure and health impact evaluation based on simultaneous measurement of indoor and ambient $\mathrm{PM}_{2.5}$ in Haidian, Beijing. Environ. Pollut. 2017, 220, 704-712. [CrossRef] [PubMed]

182. Sharma, D.; Jain, S. Impact of intervention of biomass cookstove technologies and kitchen characteristics on indoor air quality and human exposure in rural settings of India. Environ. Int. 2019, 123, 240-255. [CrossRef] [PubMed] 
183. Tong, X.; Ho, J.M.W.; Li, Z.; Lui, K.H.; Kwok, T.C.; Tsoi, K.K.; Ho, K.F. Prediction model for air particulate matter levels in the households of elderly individuals in Hong Kong. Sci. Total Environ. 2020, 717, 135323. [CrossRef] [PubMed]

184. Amoatey, P.; Omidvarborna, H.; Baawain, M.S.; Al-Mamun, A. Impact of building ventilation systems and habitual indoor incense burning on SARS-CoV-2 virus transmissions in Middle Eastern countries. Sci. Total Environ. 2020, 733, 139356. [CrossRef] [PubMed]

185. ABI. How Do Smart Homes Fit Into Smart Cities? 2020. Available online: https://www.intechnologysmartcities.com/blog/howdo-smart-homes-fit-into-smart-cities (accessed on 30 July 2020).

186. MyGlobalHome, Live Life Your Way. Available online: http://www.myglobalhome.co/ (accessed on 21 March 2021). 